\title{
Bismuth Triflate-catalyzed Three-component Mannich-type Reaction
}

\author{
Thierry Ollevier* and Etienne Nadeau \\ Département de chimie, Université Laval, Québec (Québec), Canada G1K 7 P4 \\ thierry.ollevier@chm.ulaval.ca
}

\section{Supporting Information}

\section{Contents:}

Experimental Section

S-2 to S-9

Copies of ${ }^{1} \mathrm{H}$ NMR and ${ }^{13} \mathrm{C}$ NMR spectra for compounds 4a-s

S-10 to S-46 


\section{Experimental section}

General: Infrared spectra were recorded on a FT infrared spectrometer and are reported in reciprocal centimeters $\left(\mathrm{cm}^{-1}\right),{ }^{1} \mathrm{H},{ }^{13} \mathrm{C}$, and ${ }^{19} \mathrm{~F}$ NMR spectra were recorded on a $400 \mathrm{MHz}$ magnetic resonance spectrometer in $\mathrm{CDCl}_{3}$. For ${ }^{1} \mathrm{H} \mathrm{NMR}$, tetramethylsilane (TMS) served as internal standard $(\delta=0)$ and data are reported as follows: chemical shift, integration, multiplicity ( $\mathrm{s}=$ singlet, $\mathrm{d}=$ doublet, $\mathrm{t}=$ triplet, $\mathrm{q}=$ quartet, $\mathrm{m}=$ multiplet, and $\mathrm{br}=$ broad), and coupling constant in $\mathrm{Hz}$. For ${ }^{13} \mathrm{C} \mathrm{NMR}, \mathrm{CDCl}_{3}$ was used as internal standard $(\delta=77.0)$ and spectra were obtained with complete proton decoupling. For ${ }^{19} \mathrm{~F} \mathrm{NMR}, \mathrm{CFCl}_{3}$ was used as internal standard $(\delta$ $=0)$. Column chromatography was performed on silica gel (230-400 mesh) and analytical thinlayer chromatography was carried out using $250 \mu \mathrm{m}$ commercial silica gel plates. Visualization of the developed chromatogram was performed by UV absorbance or aqueous potassium permanganate. All glassware was stored in the oven and flame-dried prior to use under an inert atmosphere of argon. Acetonitrile was distilled from calcium hydride $\left(\mathrm{CaH}_{2}\right)$.

Typical Procedure for the Bismuth-Catalysed Three-component Reaction: Under an inert atmosphere of argon, the silyl enol ether $(2.4 \mathrm{mmol})$ in $2 \mathrm{~mL}$ of dry acetonitrile was added in one portion to a solution of bismuth triflate $(0.02 \mathrm{mmol})$, the aldehyde $(2 \mathrm{mmol})$, and the aniline $(2$ $\mathrm{mmol}$ ) in $2 \mathrm{~mL}$ of dry acetonitrile. The mixture was stirred at room temperature until the reaction was completed as indicated by TLC. The reaction was quenched with water and extracted with diethyl ether. The organic phase was washed with water and saturated aqueous sodium chloride, dried over magnesium sulfate, and concentrated under vacuum (rotary evaporator). The crude product was filtered under glass frit and washed with hexane (4a-c, 4e-n) or diethyl ether (4s). 4d and 4o-r were purified by silica gel chromatography (hexane/ethyl acetate). $\beta$-amino ketones $\mathbf{4 a -}$ g, 4i-p and $4 \mathbf{s}$ accord exactly with those that have been previously reported in the literature.

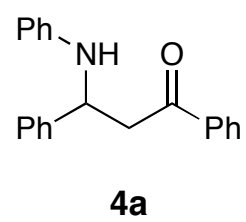

1,3-Diphenyl-3-( $N$-phenylamino)propan-1-one (4a): ${ }^{1,2}$ Yield: $89 \%$; white solid; m.p. $170-172{ }^{\circ} \mathrm{C} ; R_{\mathrm{f}} 0.51$ (hexane/ethyl acetate $\left.=4: 1\right)$; IR $(\mathrm{KBr}): v=3385,1671 ;{ }^{1} \mathrm{H} \mathrm{NMR}\left(\mathrm{CDCl}_{3}\right): \delta$ $=7.90(2 \mathrm{H}, \mathrm{d}, J=7.2 \mathrm{~Hz}), 7.56(1 \mathrm{H}, \mathrm{t}, J=7.3 \mathrm{~Hz}), 7.42-7.46(4 \mathrm{H}, \mathrm{m}), 7.32(2 \mathrm{H}, \mathrm{t}, J=7.3 \mathrm{~Hz})$, $7.21-7.25(1 \mathrm{H}, \mathrm{m}), 7.09(2 \mathrm{H}, \mathrm{t}, J=7.9 \mathrm{~Hz}), 6.69(1 \mathrm{H}, \mathrm{t}, J=7.3 \mathrm{~Hz}), 6.59(2 \mathrm{H}, \mathrm{d}, J=8.0 \mathrm{~Hz})$, $5.00(1 \mathrm{H}, \mathrm{dd}, J=7.2,5.7 \mathrm{~Hz}), 3.54(1 \mathrm{H}, \mathrm{dd}, J=16.1,5.4 \mathrm{~Hz}), 3.47(1 \mathrm{H}, \mathrm{dd}, J=16.1,7.7 \mathrm{~Hz})$;

${ }^{13} \mathrm{C}$ NMR $\left(\mathrm{CDCl}_{3}\right): \delta=198.4,146.8,142.9,136.9,133.7,129.3,129.0,128.9,128.4,127.6$, $126.7,118.3,114.3,55.3,46.4$.

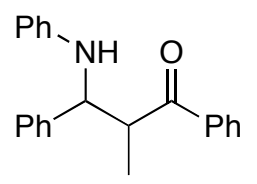


2-Methyl-1,3-diphenyl-3-( $N$-phenylamino)propan-1-one (4b): ${ }^{1-3}$ Yield: $73 \%$; syn/anti $=50 / 50$; yellowish solid; m.p. $115-117^{\circ} \mathrm{C} ; R_{\mathrm{f}} 0.50$ (hexane/ethyl acetate $\left.=4: 1\right)$; IR $(\mathrm{KBr}): v=$ 3392, 1673; ${ }^{1} \mathrm{H}$ NMR $\left(\mathrm{CDCl}_{3}\right): \delta=7.92-7.95(1 \mathrm{H}, \mathrm{m}), 7.72-7.76(1 \mathrm{H}, \mathrm{m}), 7.00-7.57(10 \mathrm{H}, \mathrm{m})$, $6.62(1 \mathrm{H}, \mathrm{dt}, J=7.2,0.8 \mathrm{~Hz}), 6.55(1 \mathrm{H}, \mathrm{d}, J=8.0 \mathrm{~Hz}), 6.47(1 \mathrm{H}, \mathrm{d}, J=7.8 \mathrm{~Hz}), 4.75(\mathrm{syn}, 1 \mathrm{H}, \mathrm{d}$, $J=5.1 \mathrm{~Hz}), 4.72($ anti, $1 \mathrm{H}, \mathrm{d}, J=6.1 \mathrm{~Hz}), 3.95-4.04(1 \mathrm{H}, \mathrm{m}), 1,29$ (anti, 3H, d, $J=7.0 \mathrm{~Hz}), 1.22$ $($ syn, 3H, d, $J=6.8 \mathrm{~Hz}) ;{ }^{13} \mathrm{C} \mathrm{NMR}\left(\mathrm{CDCl}_{3}\right): \delta=204.4,202.9,147.5,147.4,142.1,141.8,137.4$, $136.5,133.6,133.5,129.4,129.2$, 129.1, 128.9, 128.8, 128.6, 128.5, 127.6, 127.13, 127.06, $117.9,117.5,114.1,113.8,61.4,59.5,47.2,46.7,17.0,11.8$.

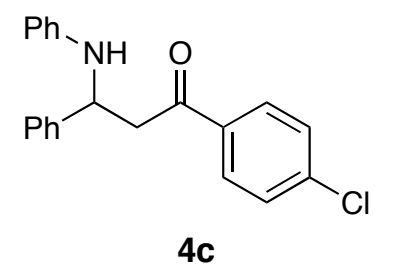

1-(4-Chlorophenyl)-3-phenyl-3-( $N$-phenylamino)propan-1-one $\quad(4 c):^{4} \quad$ Yield: $82 \%$; white solid; m.p. $123-124{ }^{\circ} \mathrm{C} ; R_{\mathrm{f}} 0.50$ (hexane/ethyl acetate $=4: 1$ ); IR $(\mathrm{KBr}): v=3378,1673 ;{ }^{1} \mathrm{H}$ $\operatorname{NMR}\left(\mathrm{CDCl}_{3}\right): \delta=7.82(2 \mathrm{H}, \mathrm{d}, J=8.6 \mathrm{~Hz}), 7.41(4 \mathrm{H}, \mathrm{t}, J=8.7 \mathrm{~Hz}), 7.31(2 \mathrm{H}, \mathrm{t}, J=7.5 \mathrm{~Hz})$, $7.23(1 \mathrm{H}, \mathrm{t}, J=7.3 \mathrm{~Hz}), 7.10(2 \mathrm{H}, \mathrm{t}, J=7.7 \mathrm{~Hz}), 6.69(1 \mathrm{H}, \mathrm{t}, J=7.2 \mathrm{~Hz}), 6.59(2 \mathrm{H}, \mathrm{t}, J=7.6 \mathrm{~Hz})$, $5.00\left(1 \mathrm{H}\right.$, br s), 3.40-3.50 $(2 \mathrm{H}, \mathrm{m}) ;{ }^{13} \mathrm{C} \mathrm{NMR}\left(\mathrm{CDCl}_{3}\right): \delta=197.2,146.6,142.6,140.1,135.2$, $129.8,129.4,129.2,129.1,127.7,126.7,118.5,114.4,55.3,46.2$.

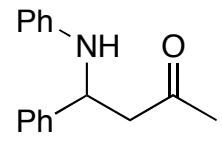

4d

4-Phenyl-4-( $N$-phenylamino)butan-2-one (4d): ${ }^{4,5}$ Yield: $42 \%$; white solid; m.p. 90-92 ${ }^{\circ} \mathrm{C} ; R_{\mathrm{f}} 0.26$ (hexane/ethyl acetate $\left.=4: 1\right)$; IR $(\mathrm{KBr}): v=3407,3370,1708 ;{ }^{1} \mathrm{H} \mathrm{NMR}\left(\mathrm{CDCl}_{3}\right): \delta=$ 7.21-7.37 (5H, m), $7.09(2 \mathrm{H}, \mathrm{t}, J=7.7 \mathrm{~Hz}), 6.68(1 \mathrm{H}, \mathrm{t}, J=7.3 \mathrm{~Hz}), 6.56(2 \mathrm{H}, \mathrm{d}, J=8.2 \mathrm{~Hz})$, $4.84(1 \mathrm{H}, \mathrm{t}, J=6.4 \mathrm{~Hz}), 2.94(2 \mathrm{H}, \mathrm{d}, J=6.4 \mathrm{~Hz}), 2.09(3 \mathrm{H}, \mathrm{s}) ;{ }^{13} \mathrm{C} \mathrm{NMR}\left(\mathrm{CDCl}_{3}\right): \delta=207.3$, $146.7,142.5,129.4,129.0,127.6,126.5,118.3,114.2,54.8,51.3,31.0$. During silica gel purification, the crude 4d undergoes a second Mannich-type reaction and gives the following cyclic adduct 4d'.

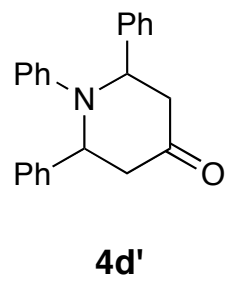

1,2,6-Triphenylpiperidin-4-one (4d'): ${ }^{6}$ Yield: $24 \%$; $\mathrm{dr}=52 / 48$; yellowish solid; m.p. $193-195{ }^{\circ} \mathrm{C} ; R_{\mathrm{f}}=0.39$ (hexane/ethyl acetate $\left.=4: 1\right)$; IR $(\mathrm{KBr}): v=1723 ;{ }^{1} \mathrm{H} \mathrm{NMR}\left(\mathrm{CDCl}_{3}\right): \delta=$ 6.49-7.56 (15H, m), 5.46 (1 diastereoisomer, $2 \mathrm{H}, \mathrm{d}, J=4.5 \mathrm{~Hz}), 4.85$ (1 diastereoisomer, $2 \mathrm{H}$, dd, 
$J=9.3,3.4 \mathrm{~Hz}), 3.22(1$ diastereoisomer, $2 \mathrm{H}, \mathrm{dd}, J=17.4,6.4 \mathrm{~Hz}), 2.96-3.02(2$ diastereoisomers, $4 \mathrm{H}, \mathrm{m}), 2.82$ (1 diastereoisomer, $2 \mathrm{H}, \mathrm{dd}, J=15.8,3.3 \mathrm{~Hz}$ ); HRMS: Calcd for $\mathrm{C}_{23} \mathrm{H}_{21} \mathrm{NO}(\mathrm{M}+)$ 327.1623, found 327.1630 .

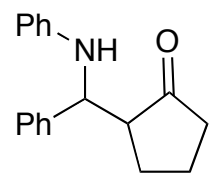

$4 e$

2-(Phenyl- $N$-phenylaminomethyl)cyclopentanone (4e): ${ }^{2}$ Yield: $80 \%$; syn/ant $i=68 / 32$; yellow solid; m.p. $112-114{ }^{\circ} \mathrm{C} ; R_{\mathrm{f}} 0.35$ (hexane/ethyl acetate $=4: 1$ ); $\mathrm{IR}(\mathrm{KBr}): v=3377,1724 ;{ }^{1} \mathrm{H}$ NMR $\left(\mathrm{CDCl}_{3}\right): \delta=7.19-7.40(5 \mathrm{H}, \mathrm{m}), 7.05-7.10(2 \mathrm{H}, \mathrm{m}), 6.57-6,73(3 \mathrm{H}, \mathrm{m}), 4.75(\operatorname{syn}, 1 \mathrm{H}, \mathrm{d}, J$ $=4.3 \mathrm{~Hz}$ ), 4.52 (anti, $1 \mathrm{H}, J=7.4 \mathrm{~Hz}), 2.63-2.73(1 \mathrm{H}, \mathrm{m}), 2.24-2.38(1 \mathrm{H}, \mathrm{m}), 2.07-2.15(1 \mathrm{H}, \mathrm{m})$, 1.66-1.94 $(4 \mathrm{H}, \mathrm{m}) ;{ }^{13} \mathrm{C}$ NMR $\left(\mathrm{CDCl}_{3}\right): \delta=220.8,219.6,147.8,147.0,141.9,141.1,129.3,129.2$, $128.9,128.8,127.7,127.6,127.4,118.1,117.7,114.4,113.9,59.3,57.9,54.3,53.6,40.0,39.5$, 26.9, 26.1, 20.9, 20.7.<smiles>O=C1CCCCC1C(Nc1ccccc1)c1ccccc1</smiles>

$4 f$

2-(Phenyl- $N$-phenylaminomethyl)cyclohexanone (4f): ${ }^{1,3}$ Yield: $81 \%$; syn/anti $=39 / 61$; white solid; m.p. $115-116{ }^{\circ} \mathrm{C} ; R_{\mathrm{f}} 0.39$ (hexane/ethyl acetate $=4: 1$ ); IR $(\mathrm{KBr}): v=3383,1696 ;{ }^{1} \mathrm{H}$ $\operatorname{NMR}\left(\mathrm{CDCl}_{3}\right): \delta=7.18-7.38(5 \mathrm{H}, \mathrm{m}), 7.03-7.09(2 \mathrm{H}, \mathrm{m}), 6.59-6.66(1 \mathrm{H}, \mathrm{m}), 6.51-6.56(2 \mathrm{H}, \mathrm{m})$, 4.80 (anti, $1 \mathrm{H}, \mathrm{d}, J=4.5 \mathrm{~Hz}), 4.62(\mathrm{syn}, 1 \mathrm{H}, \mathrm{d}, J=7.0 \mathrm{~Hz}) ; 4.59(1 \mathrm{H}, \mathrm{br} \mathrm{s}), 2.73-2.81(1 \mathrm{H}, \mathrm{m})$, 2.25-2.44 (2H, m), 1.52-2.08 (6H, m); ${ }^{13} \mathrm{C}$ NMR $\left(\mathrm{CDCl}_{3}\right): \delta=213.2,211.6,147.7,147.2,141.74$, $141.71,129.3,129.2,128.7,128.6,127.7,127.5,127.4,127.2,117.9,114.3,114.0,58.4,57.6$, $57.5,56.8,42.6,42.0,31.5,28.9,28.1,27.3,25.1,23.9$.<smiles>O=C(CC(Nc1ccccc1)c1ccccc1)c1ccccc1</smiles>

3-(4-Chlorophenyl)-1-phenyl-3-( $N$-phenylamino)propan-1-one $\quad(4 g):^{1} \quad$ Yield: $82 \%$; white solid; m.p. $114-115{ }^{\circ} \mathrm{C} ; R_{\mathrm{f}} 0.39$ (hexane/ethyl acetate $=4: 1$ ); IR $(\mathrm{KBr}): v=3393,1669 ;{ }^{1} \mathrm{H}$ $\operatorname{NMR}\left(\mathrm{CDCl}_{3}\right): \delta=7.88-7.90(2 \mathrm{H}, \mathrm{m}), 7.57(1 \mathrm{H}, \mathrm{t}, J=7.3 \mathrm{~Hz}), 7.44(2 \mathrm{H}, \mathrm{t}, J=7.8 \mathrm{~Hz}), 7.38(2 \mathrm{H}$, $\mathrm{d}, J=8.6 \mathrm{~Hz}), 7.26-7.29(2 \mathrm{H}, \mathrm{m}), 7.10(2 \mathrm{H}, \mathrm{dd}, J=7.5,8.3 \mathrm{~Hz}), 6.70(1 \mathrm{H}, \mathrm{t}, J=7.4 \mathrm{~Hz}), 6.55$ $(2 \mathrm{H}, \mathrm{d}, J=7.8 \mathrm{~Hz}), 4.98(1 \mathrm{H}, \mathrm{t}, J=6.4 \mathrm{~Hz}), 3.49(1 \mathrm{H}, \mathrm{dd}, J=16.5,5.6 \mathrm{~Hz}), 3.44(1 \mathrm{H}, \mathrm{dd}, J=$ 
16.5, 7.2 Hz); ${ }^{13} \mathrm{C}$ NMR $\left(\mathrm{CDCl}_{3}\right): \delta=198.1,146.6,141.5,136.7,133.8,133.2,129.4,129.2$, $129.0,128.4,128.1,118.5,114.3,54.6,46.2$.<smiles>O=C(CC(Nc1ccccc1)c1ccccc1)c1ccccc1</smiles>

3-[4-(Trifluoromethyl)phenyl]-1-phenyl-3-( $N$-phenylamino)propan-1-one (4h): Yield: $88 \%$; white solid; m.p. $144-145{ }^{\circ} \mathrm{C} ; R_{\mathrm{f}} 0.39$ (hexane/ethyl acetate $\left.=4: 1\right)$; $\mathrm{IR}(\mathrm{KBr}): v=3403$, $1671 ;{ }^{1} \mathrm{H} \mathrm{NMR}\left(\mathrm{CDCl}_{3}\right): \delta=7.89(2 \mathrm{H}, \mathrm{d}, J=7.4 \mathrm{~Hz}), 7.55-7.59(5 \mathrm{H}, \mathrm{m}), 7.44(2 \mathrm{H}, \mathrm{t}, J=7.7 \mathrm{~Hz})$, $7.11(2 \mathrm{H}, \mathrm{t}, J=7.9 \mathrm{~Hz}), 6.71(1 \mathrm{H}, \mathrm{t}, J=7.3 \mathrm{~Hz}), 6.56(2 \mathrm{H}, \mathrm{d}, J=7.8 \mathrm{~Hz}), 5.06(1 \mathrm{H}, \mathrm{t}, J=6.3$ $\mathrm{Hz}), 3.52(1 \mathrm{H}, \mathrm{d}, J=2.5 \mathrm{~Hz}), 3.50(1 \mathrm{H}, \mathrm{d}, J=3.7 \mathrm{~Hz}) ;{ }^{13} \mathrm{C} \mathrm{NMR}\left(\mathrm{CDCl}_{3}\right): \delta=197.9,147.3$, $146.7,136.7,133.9,129.8(\mathrm{q}, J=32.2 \mathrm{~Hz}), 129.5,129.0,128.6,128.4,127.1,126.0$ (q, $J=3.8$ $\mathrm{Hz}), 124.3(\mathrm{q}, J=270 \mathrm{~Hz}), 118.5,114.2,54.6,46.2 ;{ }^{19} \mathrm{~F}$ NMR $\left(\mathrm{CDCl}_{3}\right): \delta=-62.94$; HRMS: Calcd for $\mathrm{C}_{22} \mathrm{H}_{18} \mathrm{~F}_{3} \mathrm{NO}(\mathrm{M}+)$ 369.1340, found 369.1336.<smiles>O=C(CC(Nc1ccccc1)c1ccccc1)c1ccccc1</smiles>

3-(4-Nitrophenyl)-1-phenyl-3-( $N$-phenylamino)propan-1-one (4i): ${ }^{1}$ Yield: 87\%; yellow solid; m.p. $121-122{ }^{\circ} \mathrm{C} ; R_{\mathrm{f}} 0.21$ (hexane/ethyl acetate $=4: 1$ ); IR $(\mathrm{KBr}): v=3403,1671 ;{ }^{1} \mathrm{H}$ NMR $\left(\mathrm{CDCl}_{3}\right): \delta=8.16(2 \mathrm{H}, \mathrm{d}, J=8.8 \mathrm{~Hz}), 7.87-7.90(2 \mathrm{H}, \mathrm{m}), 7.65(2 \mathrm{H}, \mathrm{d}, J=8.6 \mathrm{~Hz}), 7.58(1 \mathrm{H}, \mathrm{tt}, J$ $=7.4,1.4 \mathrm{~Hz}), 7.45(2 \mathrm{H}, \mathrm{t}, J=7.7 \mathrm{~Hz}), 7.12(2 \mathrm{H}, \mathrm{dd}, J=8.4,7.4 \mathrm{~Hz}), 6.76(1 \mathrm{H}, \mathrm{t}, J=7.4 \mathrm{~Hz})$, $6.59(2 \mathrm{H}, \mathrm{d}, J=8.0 \mathrm{~Hz}), 5.12(1 \mathrm{H}, \mathrm{t}, J=6.2 \mathrm{~Hz}), 3.58(2 \mathrm{H}, \mathrm{d}, J=6.2 \mathrm{~Hz}) ;{ }^{13} \mathrm{C} \mathrm{NMR}\left(\mathrm{CDCl}_{3}\right): \delta$ $=197.5,151.0,147.4,146.5,136.5,134.1,129.5,129.1,128.4,127.8,124.3,118.7,114.1,54.4$, 45.9.

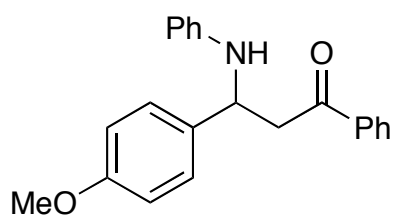

$4 \mathbf{j}$

3-(4-Methoxyphenyl)-1-phenyl-3-( $N$-phenylamino)propan-1-one $\quad(4 \mathbf{j}):^{7}$ Yield: $86 \%$; white solid; m.p. $150-151{ }^{\circ} \mathrm{C} ; R_{\mathrm{f}} 0.32$ (hexane/ethyl acetate $=4: 1$ ); $\mathrm{IR}(\mathrm{KBr}): v=3377,1667 ;{ }^{1} \mathrm{H}$ NMR $\left(\mathrm{CDCl}_{3}\right): \delta=7.89-7.91(2 \mathrm{H}, \mathrm{m}), 7.54-7.57(1 \mathrm{H}, \mathrm{m}), 7.44(2 \mathrm{H}, \mathrm{t}, J=7.7 \mathrm{~Hz}), 7.35(2 \mathrm{H}, \mathrm{d}, J$ $=8.6 \mathrm{~Hz}), 7.07-7.11(2 \mathrm{H}, \mathrm{m}), 6.83-6.86(2 \mathrm{H}, \mathrm{m}), 6.68(1 \mathrm{H}, \mathrm{t}, J=7.3 \mathrm{~Hz}), 6.59(2 \mathrm{H}, \mathrm{d}, J=7.8$ $\mathrm{Hz}), 4.96(1 \mathrm{H}, \mathrm{t}, J=6.4 \mathrm{~Hz}), 3.76(3 \mathrm{H}, \mathrm{s}), 3.51(1 \mathrm{H}, \mathrm{dd}, J=16.2,5.6 \mathrm{~Hz}), 3.44(1 \mathrm{H}, \mathrm{dd}, J=16.2$, 
$7.4 \mathrm{~Hz}) ;{ }^{13} \mathrm{C} \mathrm{NMR}\left(\mathrm{CDCl}_{3}\right): \delta=198.6,159.0,147.0,136.9,135.0,133.6,129.3,128.9,128.4$, $127.7,118.1,114.4,114.2,55.5,54.6,46.5$.

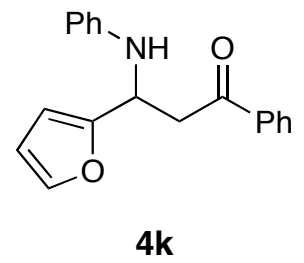

3-(Furan-2-yl)-1-phenyl-3-( $N$-phenylamino)propan-1-one (4k): ${ }^{1,4}$ Yield: $76 \%$; white solid; m.p. $105-106{ }^{\circ} \mathrm{C} ; R_{\mathrm{f}} 0.42$ (hexane/ethyl acetate $=4: 1$ ); IR $(\mathrm{KBr}): v=3351,1672 ;{ }^{1} \mathrm{H}$ NMR $\left(\mathrm{CDCl}_{3}\right): \delta=7.92(2 \mathrm{H}, \mathrm{d}, J=7.6 \mathrm{~Hz}), 7.56(1 \mathrm{H}, \mathrm{t}, J=7.3 \mathrm{~Hz}), 7.44(2 \mathrm{H}, \mathrm{t}, 7.6 \mathrm{~Hz}), 7.30(1 \mathrm{H}, \mathrm{s})$, $7.16(2 \mathrm{H}, \mathrm{t}, J=7.8 \mathrm{~Hz}), 6.70-6.76(3 \mathrm{H}, \mathrm{m}), 6.20-6.27(2 \mathrm{H}, \mathrm{m}), 5.22(1 \mathrm{H}, \mathrm{t}, J=6.1 \mathrm{~Hz}), 3.59(2 \mathrm{H}$, $\mathrm{d}, J=6.1 \mathrm{~Hz}) ;{ }^{13} \mathrm{C}$ NMR $\left(\mathrm{CDCl}_{3}\right): \delta=198.1,155.0,146.6,141.9,137.0,133.6,129.5,128.9$, $128.4,118.7,114.3,110.6,106.8,49.0,42.5$.

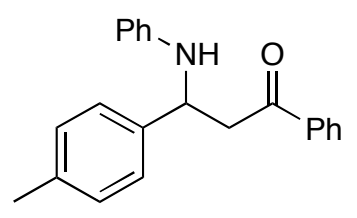

4

1-Phenyl-3-( $N$-phenylamino)-3-p-tolylpropan-1-one (41): ${ }^{1}$ Yield: $82 \%$; white solid; m.p. $139-141{ }^{\circ} \mathrm{C} ; R_{\mathrm{f}} 0.47$ (hexane/ethyl acetate $=4: 1$ ); IR $(\mathrm{KBr}): v=3388,1669 ;{ }^{1} \mathrm{H}$ NMR $\left(\mathrm{CDCl}_{3}\right): \delta=7.89-7.91(2 \mathrm{H}, \mathrm{m}), 7.54-7.57(1 \mathrm{H}, \mathrm{m}), 7.43(2 \mathrm{H}, \mathrm{t}, J=7.7 \mathrm{~Hz}), 7.33(2 \mathrm{H}, \mathrm{d}, J=8.2$ $\mathrm{Hz}), 7.08-7.13(4 \mathrm{H}, \mathrm{m}), 6.72(1 \mathrm{H}, \mathrm{t}, J=7.3 \mathrm{~Hz}), 6.64(2 \mathrm{H}, \mathrm{d}, J=8.0 \mathrm{~Hz}), 4.98(1 \mathrm{H}, \mathrm{t}, J=6.4$ $\mathrm{Hz}), 3.48-3.60(2 \mathrm{H}, \mathrm{m}), 2.30(3 \mathrm{H}, \mathrm{s}) ;{ }^{13} \mathrm{C} \mathrm{NMR}\left(\mathrm{CDCl}_{3}\right): \delta=198.5,147.0,140.0,137.2,136.9$, $133.6,129.7,129.3,128.9,128.4,126.5,118.1,114.2,54.9,46.5,21.3$.

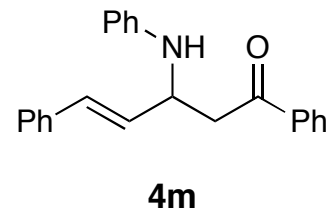

(E)-1,5-Diphenyl-3-( $N$-phenylamino)pent-4-en-1-one $(4 \mathrm{~m}):^{1}$ Yield: $72 \%$; yellowish solid; m.p. 121-123 ${ }^{\circ} \mathrm{C} ; R_{\mathrm{f}} 0.41$ (hexane/ethyl acetate $=4: 1$ ); IR $(\mathrm{KBr}): v=3390,3369,1671 ;{ }^{1} \mathrm{H}$ NMR $\left(\mathrm{CDCl}_{3}\right): \delta=7.95(2 \mathrm{H}, \mathrm{d}, J=7.2 \mathrm{~Hz}), 7.58(1 \mathrm{H}, \mathrm{t}, J=7.3 \mathrm{~Hz}), 7.46(2 \mathrm{H}, \mathrm{t}, J=7.7 \mathrm{~Hz})$, 7.17-7.32 (7H, m), 6.76-6.81 (3H, m), $6.62(1 \mathrm{H}, \mathrm{d}, J=16.0 \mathrm{~Hz}), 6.34(1 \mathrm{H}, \mathrm{dd}, J=16.0,6.4 \mathrm{~Hz})$, $4.68(1 \mathrm{H}, \mathrm{dd}, J=11.9,6.1 \mathrm{~Hz}), 3.41-3.52(2 \mathrm{H}, \mathrm{m}) ;{ }^{13} \mathrm{C} \mathrm{NMR}\left(\mathrm{CDCl}_{3}\right): \delta=198.6,146.5,137.0$, $136.8,133.7,131.3,130.3,129.5,128.9,128.7,128.4,127.8,126.7,118.7,114.6,53.0,44.0$. 


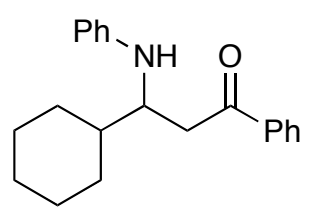

$4 n$

3-Cyclohexyl-1-phenyl-3-( $N$-phenylamino)propan-1-one $\quad(4 \mathbf{n}):^{1}$ Yield: $77 \%$; white solid; m.p. $111-112{ }^{\circ} \mathrm{C} ; R_{\mathrm{f}} 0.60$ (hexane/ethyl acetate $\left.=4: 1\right)$; IR $(\mathrm{KBr}): v=3396,1678 ;{ }^{1} \mathrm{H} \mathrm{NMR}$ $\left(\mathrm{CDCl}_{3}\right): \delta=7.90-7.92(2 \mathrm{H}, \mathrm{m}), 7.53-7.57(1 \mathrm{H}, \mathrm{m}), 7.44(2 \mathrm{H}, \mathrm{t}, J=7.6 \mathrm{~Hz}), 7.11-7.15(2 \mathrm{H}, \mathrm{m})$, 6.61-6.67 (3H, m), $3.92(1 \mathrm{H}, \mathrm{q}, J=5.7 \mathrm{~Hz}), 3.24(1 \mathrm{H}, \mathrm{dd}, J=16.7,5.8 \mathrm{~Hz}), 3.12(1 \mathrm{H}, \mathrm{dd}, J=$ 16.7, $5.7 \mathrm{~Hz}), 1.94(1 \mathrm{H}, \mathrm{d}, J=12.3 \mathrm{~Hz}), 1.66-1.78(5 \mathrm{H}, \mathrm{m}), 1.00-1.27(5 \mathrm{H}, \mathrm{m}) ;{ }^{13} \mathrm{C} \mathrm{NMR}$ $\left(\mathrm{CDCl}_{3}\right): \delta=199.7,147.9,137.4,133.3,129.6,128.9,128.3,117.3,113.5,55.0,42.3,40.6,30.1$, 29.7, 26.7, 26.6.

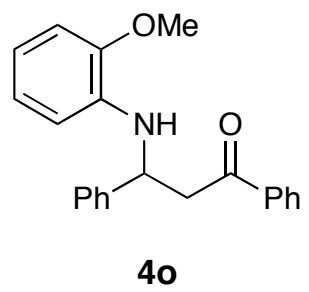

3-[N-(2-Methoxyphenylamino)]-1,3-diphenylpropan-1-one (40): ${ }^{8}$ Yield: $65 \%$; white solid; m.p. $113-114{ }^{\circ} \mathrm{C} ; R_{\mathrm{f}} 0.43$ (hexane/ethyl acetate $=4: 1$ ); IR $(\mathrm{KBr}): v=3413,1673 ;{ }^{1} \mathrm{H}$ NMR $\left(\mathrm{CDCl}_{3}\right): \delta=7.90-7.92(2 \mathrm{H}, \mathrm{m}), 7.53-7.57(1 \mathrm{H}, \mathrm{m}), 7.41-7.46(4 \mathrm{H}, \mathrm{m}), 7.31(2 \mathrm{H}, \mathrm{t}, J=7.5 \mathrm{~Hz})$, 7.20-7.25 (1H, m), 6.64-6.77 (3H, m), $6.51(1 \mathrm{H}, \mathrm{d}, J=6.8 \mathrm{~Hz}), 5.06(1 \mathrm{H}, \mathrm{t}, J=6.5 \mathrm{~Hz}), 3.85$ $(3 \mathrm{H}, \mathrm{s}), 3.52-3.83(2 \mathrm{H}, \mathrm{m}) ;{ }^{13} \mathrm{C} \mathrm{NMR}\left(\mathrm{CDCl}_{3}\right): \delta=198.1,147.3,143.2,137.0,136.8,133.5$, $129.0,128.9,128.4,127.6,126.7,121.3,117.3,111.8,109.7,55.7,54.7,46.8$.

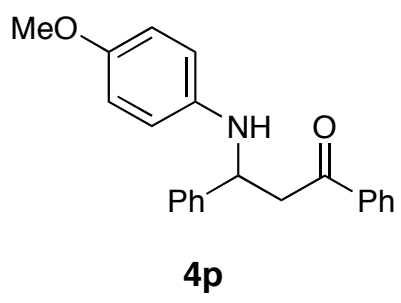

3-[N-(4-Methoxyphenylamino)]-1,3-diphenylpropan-1-one $\quad(4 p):^{1,2} \quad$ Yield: $\quad 79 \%$; yellowish solid; m.p. $130-132{ }^{\circ} \mathrm{C} ; R_{\mathrm{f}} 0.22$ (hexane/ethyl acetate $\left.=4: 1\right)$; IR $(\mathrm{KBr}): v=3373,1667$; ${ }^{1} \mathrm{H} \operatorname{NMR}\left(\mathrm{CDCl}_{3}\right): \delta=7.89-7.92(2 \mathrm{H}, \mathrm{m}), 7.55(1 \mathrm{H}, \mathrm{tt}, J=7.4,1.6 \mathrm{~Hz}), 7.42-7.46(4 \mathrm{H}, \mathrm{m}), 7.30-$ $7.34(2 \mathrm{H}, \mathrm{m}), 7.23(1 \mathrm{H}, \mathrm{tt}, J=7.3,1.6 \mathrm{~Hz}), 6.68(2 \mathrm{H}, \mathrm{dt}, J=9.0,2.9 \mathrm{~Hz}), 6.54(2 \mathrm{H}, \mathrm{dt}, J=9.0$, $2.9 \mathrm{~Hz}), 4.92(1 \mathrm{H}, \mathrm{dd}, J=7.6,5.3 \mathrm{~Hz}), 3.68(3 \mathrm{H}, \mathrm{s}), 3.50(1 \mathrm{H}, \mathrm{dd} J=16.2,5.3 \mathrm{~Hz}), 3.42(1 \mathrm{H}$, dd, $J=16.2,7.6 \mathrm{~Hz}) ;{ }^{13} \mathrm{C} \mathrm{NMR}\left(\mathrm{CDCl}_{3}\right): \delta=198.5,152.7,143.2,141.0,136.9,133.6,129.0,128.9$, $128.4,127.6,126.7,115.8,114.9,56.2,55.9,46.5$. 


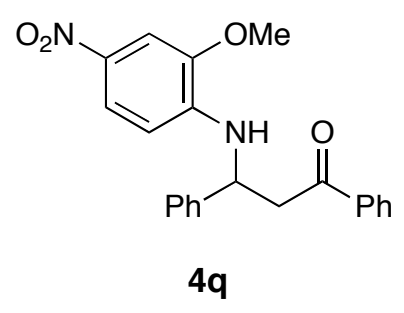

3-[N-(2-Methoxy-4-nitrophenylamino)]-1,3-diphenylpropan-1-one (4q): Yield: $85 \%$; yellow solid; m.p. $138-140{ }^{\circ} \mathrm{C} ; R_{\mathrm{f}} 0.18$ (hexane/ethyl acetate $=4: 1$ ); $\mathrm{IR}(\mathrm{KBr}): v=3393,1683 ;{ }^{1} \mathrm{H}$ NMR $\left(\mathrm{CDCl}_{3}\right): \delta=7.90-7.92(2 \mathrm{H}, \mathrm{m}), 7.74(1 \mathrm{H}, \mathrm{dd}, J=8.9,2.4 \mathrm{~Hz}), 7.61(1 \mathrm{H}, \mathrm{d}, J=2.3 \mathrm{~Hz})$, $7.58(1 \mathrm{H}, \mathrm{t}, J=7.5 \mathrm{~Hz}), 7.46(2 \mathrm{H}, \mathrm{t}, J=7.7 \mathrm{~Hz}), 7.32-7.40(4 \mathrm{H}, \mathrm{m}), 7.25-7.28(1 \mathrm{H}, \mathrm{m}), 6.37(1 \mathrm{H}$, d, $J=9.0 \mathrm{~Hz}), 5.17(1 \mathrm{H}, \mathrm{t}, J=6.3 \mathrm{~Hz}), 3.95(3 \mathrm{H}, \mathrm{s}), 3.57(1 \mathrm{H}, \mathrm{d}, J=3.3 \mathrm{~Hz}), 3.55(1 \mathrm{H}, \mathrm{d}, J=2.0$ $\mathrm{Hz}) ;{ }^{13} \mathrm{C}$ NMR $\left(\mathrm{CDCl}_{3}\right): \delta=197.5,145.7,143.1,141.6,137.8,136.7,133.9,129.3,129.0,128.4$, 128.1, 126.4, 119.7, 108.5, 104.9, 56.2, 53.9, 46.2. HRMS: Calcd for $\mathrm{C}_{22} \mathrm{H}_{20} \mathrm{~N}_{2} \mathrm{O}_{4}(\mathrm{M}+)$ 376.1423 , found 376.1419 .

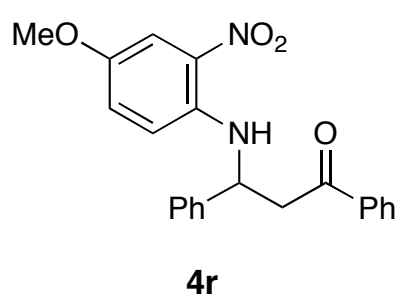

3-[N-(4-Methoxy-2-nitrophenylamino)]-1,3-diphenylpropan-1-one (4r): Yield: $88 \%$; orange solid; m.p. $106-108{ }^{\circ} \mathrm{C} ; R_{\mathrm{f}} 0.31$ (hexane/ethyl acetate $=4: 1$ ); IR $(\mathrm{KBr}): v=3352,1683 ;{ }^{1} \mathrm{H}$ NMR $\left(\mathrm{CDCl}_{3}\right): \delta=8.53(1 \mathrm{H}$, br s), 7.91-7.93 $(2 \mathrm{H}, \mathrm{m}), 7.56-7.61(2 \mathrm{H}, \mathrm{m}), 7.41-7.47(4 \mathrm{H}, \mathrm{m})$, $7.34(2 \mathrm{H}, \mathrm{t}, J=7.5 \mathrm{~Hz}), 7.24-7.28(1 \mathrm{H}, \mathrm{m}), 7.01(1 \mathrm{H}, \mathrm{dd}, J=9.4,3.1 \mathrm{~Hz}), 6.77(1 \mathrm{H}, \mathrm{d}, J=9.4$ $\mathrm{Hz}), 5.34(1 \mathrm{H}, \mathrm{t}, J=6.1 \mathrm{~Hz}), 3.75(3 \mathrm{H}, \mathrm{s}), 3.64(1 \mathrm{H}, \mathrm{dd}, J=16.9,7.5 \mathrm{~Hz}), 3.50(1 \mathrm{H}, \mathrm{dd}, J=16.9$, $5.4 \mathrm{~Hz}) ;{ }^{13} \mathrm{C}$ NMR $\left(\mathrm{CDCl}_{3}\right): \delta=196.9,150.2,142.0,140.3,136.7,133.8,131.9,129.3,129.0$, 128.4, 128.0, 127.1, 126.5, 116.7, 107.4, 56.0, 54.0, 47.1; HRMS: Calcd for $\mathrm{C}_{22} \mathrm{H}_{20} \mathrm{~N}_{2} \mathrm{O}_{4}(\mathrm{M}+)$ 376.1423 , found 376.1419 .

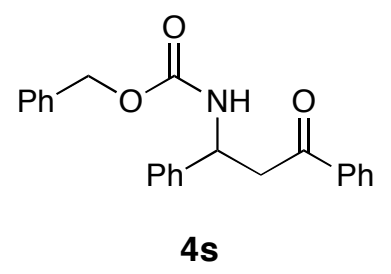

Benzyl 3-oxo-1,3-diphenylpropylcarbamate (4s): ${ }^{9}$ Yield: 49\%; white solid; m.p. 114$116{ }^{\circ} \mathrm{C} ; R_{\mathrm{f}} 0.16$ (hexane/ethyl acetate $\left.=4: 1\right)$; IR $(\mathrm{KBr}): v=3375,1719,1681 ;{ }^{1} \mathrm{H} \mathrm{NMR}\left(\mathrm{CDCl}_{3}\right)$ : $\delta=7.88(2 \mathrm{H}, \mathrm{d}, J=7.4 \mathrm{~Hz}), 7.55(1 \mathrm{H}, \mathrm{t}, J=7.5 \mathrm{~Hz}), 7.22-7.54(12 \mathrm{H}, \mathrm{m}), 5.80-5.90(1 \mathrm{H}, \mathrm{br} \mathrm{s})$, $5.33(1 \mathrm{H}, \mathrm{dd}, J=14.1,5.9 \mathrm{~Hz}), 5.11(1 \mathrm{H}, \mathrm{d}, J=12.2 \mathrm{~Hz}), 5.09(1 \mathrm{H}, \mathrm{d}, J=12.2 \mathrm{~Hz}), 3.66-3.77$ $(1 \mathrm{H}, \mathrm{m}), 3.46(1 \mathrm{H}, \mathrm{dd}, J=16.6,6.3 \mathrm{~Hz}) ;{ }^{13} \mathrm{C} \mathrm{NMR}\left(\mathrm{CDCl}_{3}\right): \delta=198.1,156.0,141.5,136.8$, $136.6,133.7,128.9,128.7,128.3,127.7,126.6,67.0,52.0,44.2$. 
(1) Akiyama, T.; Takaya, J.; Kagoshima, H. Adv. Synth. Catal. 2002, 344, 338-347.

(2) Loh, T.-P.; Liung, S. B. K. W.; Tan, K.-L.; Wei, L.-L. Tetrahedron 2000, 56, 3227-3237.

(3) Kobayashi, S.; Nagayama, S. J. Am. Chem. Soc. 1997, 119, 10049-10053.

(4) Ranu, B. C.; Samanta, S.; Guchhait, S. K. Tetrahedron 2002, 58, 983-988.

(5) Kobayashi, S.; Ishitani, H.; Nagayama, S. Synthesis 1995, 1195-1202.

(6) Kozlov, N. S.; Pak, V. D.; Nikolaev, A. D. Zhurnal Organicheskoi Khimii 1968, 4, 18421846.

(7) Yi, L.; Lei, H.; Zou, J.; Xu, X. Synthesis 1991, 717-718.

(8) Manabe, K.; Mori, Y.; Wakabayashi, T.; Nagayama, S.; Kobayashi, S. J. Am. Chem. Soc. 2000, 122, 7202-7207.

(9) Xu, L.-W.; Xia, C.-G. Tetrahedron Lett. 2004, 45, 4507-4510. 


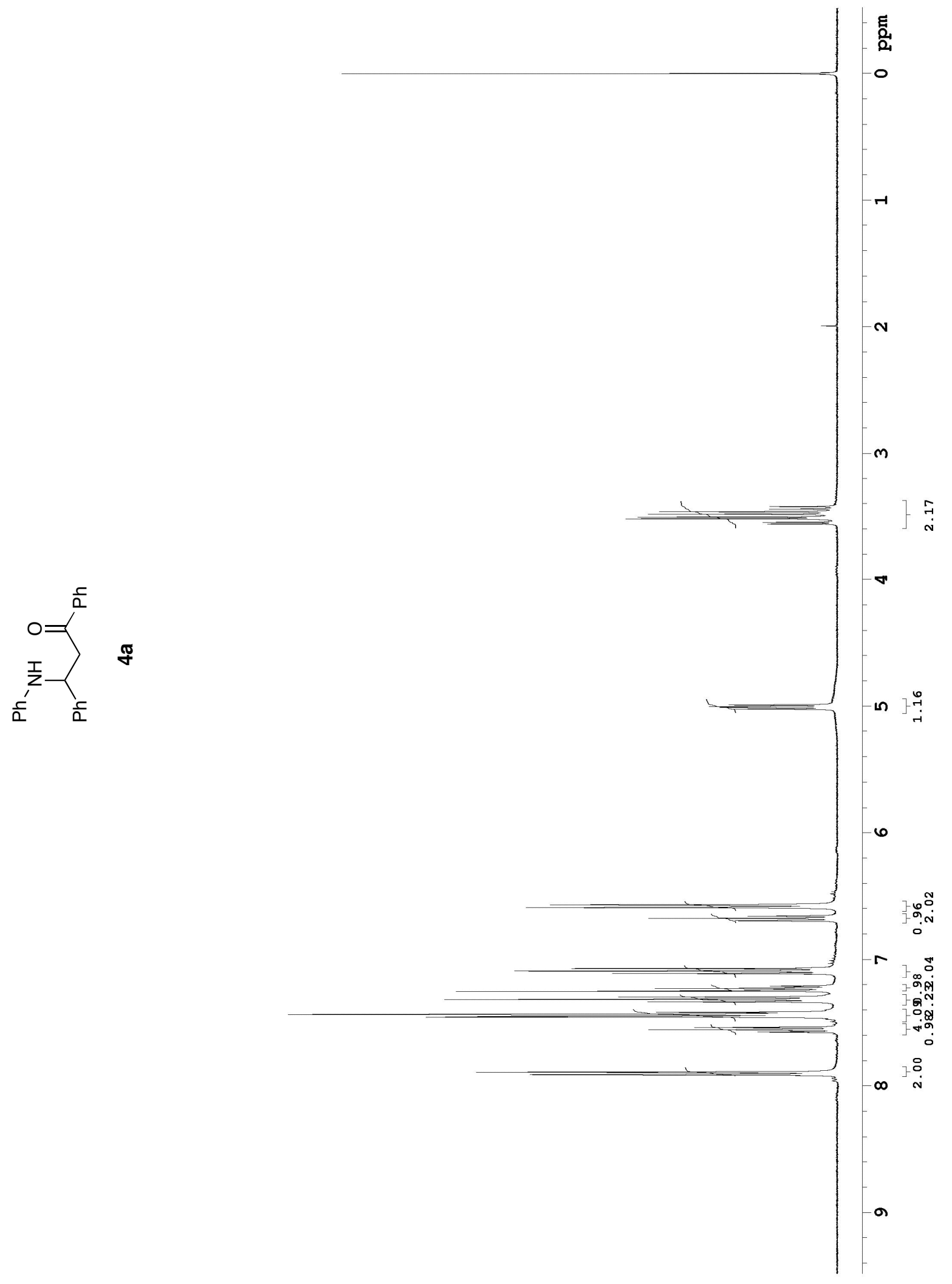

S-10 

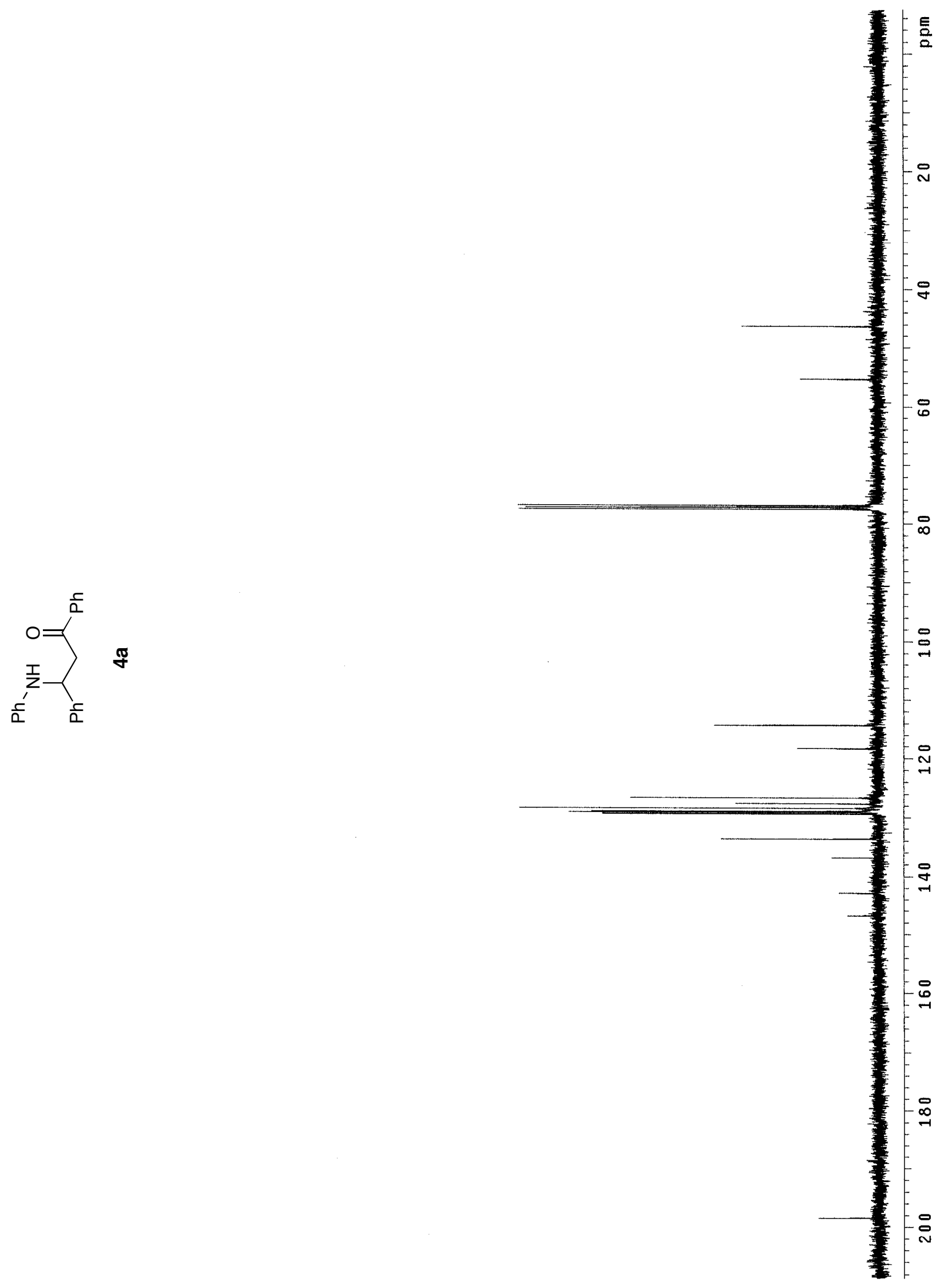

S-11 


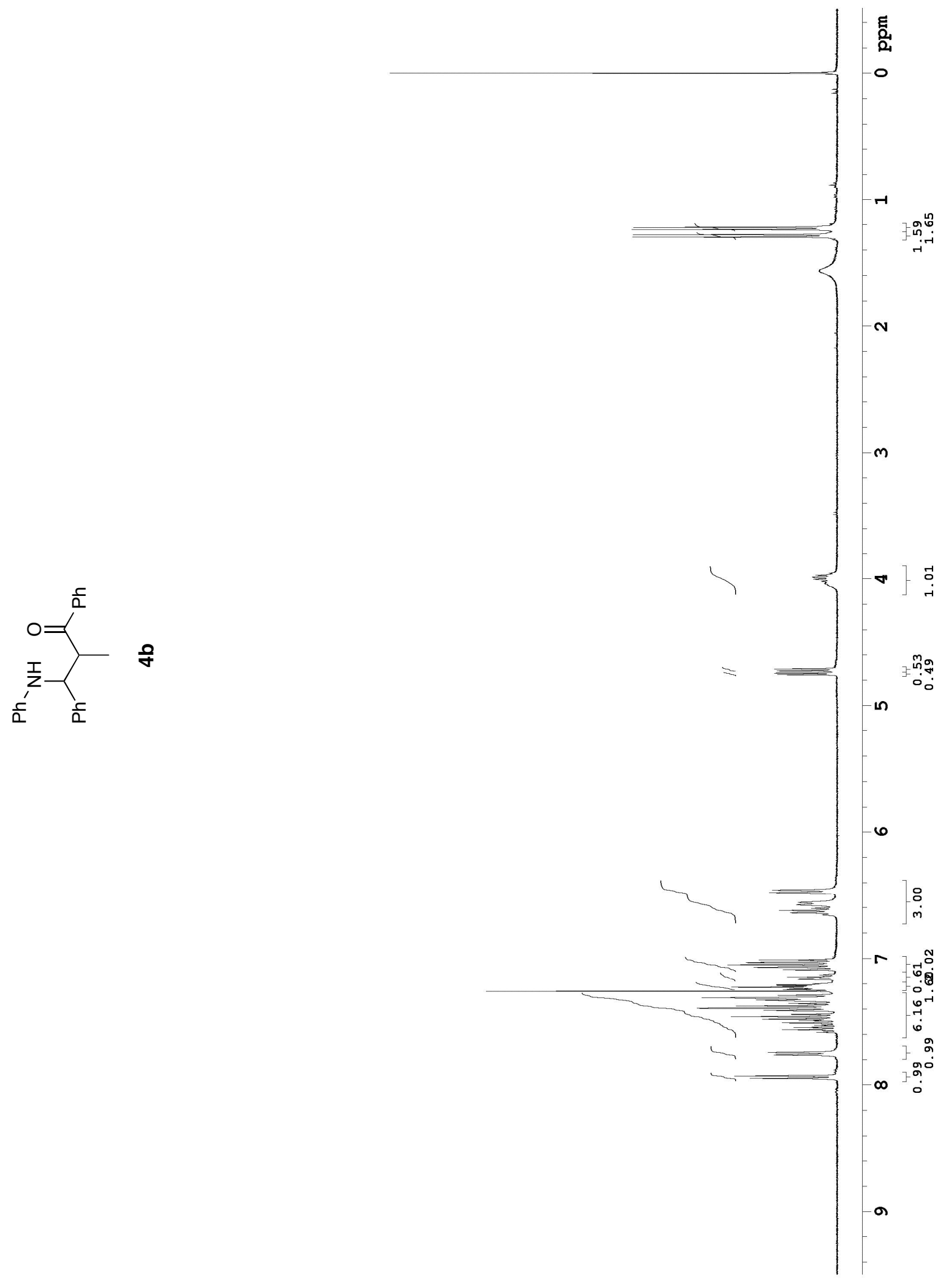

S-12 


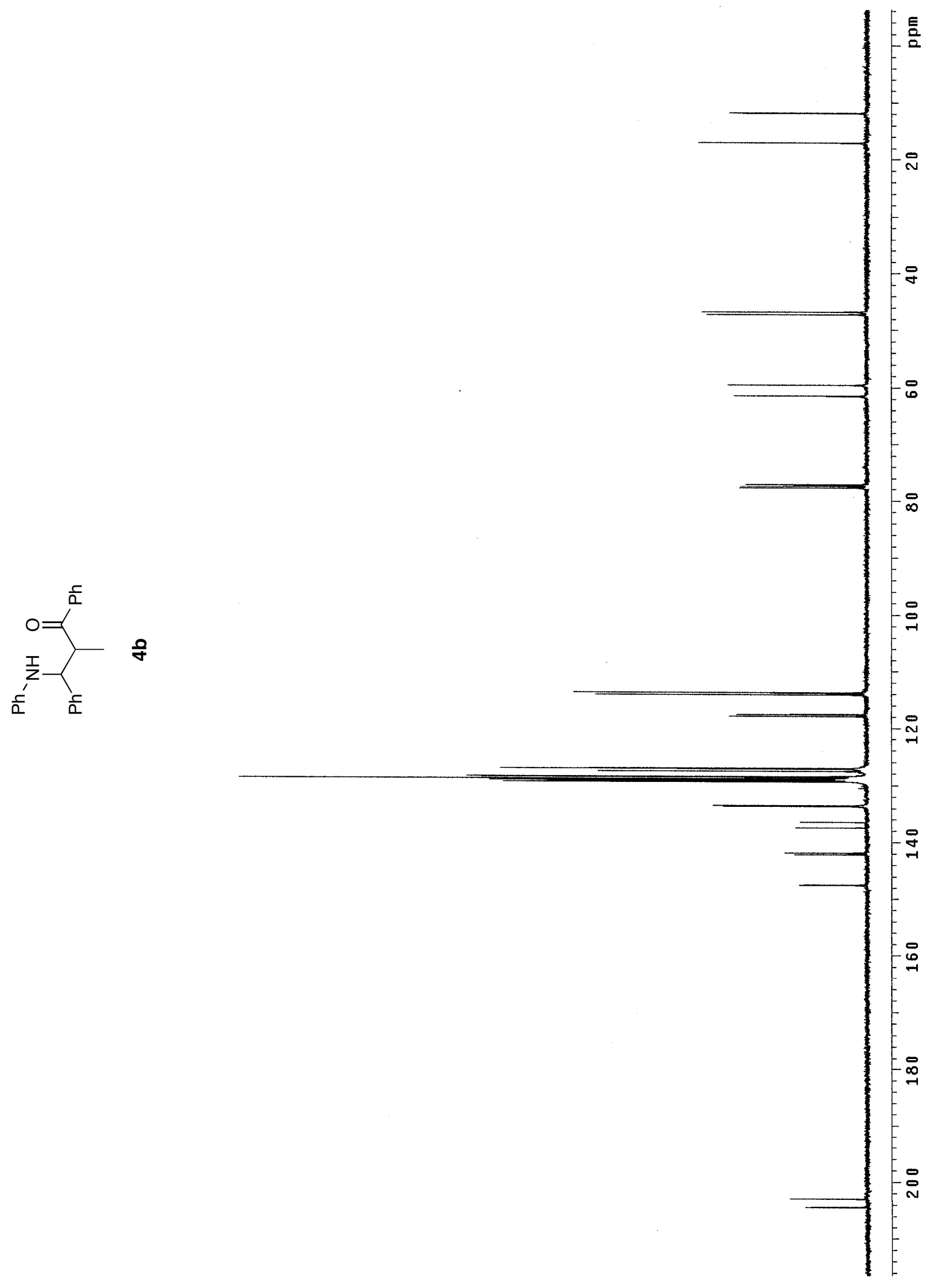

S-13 


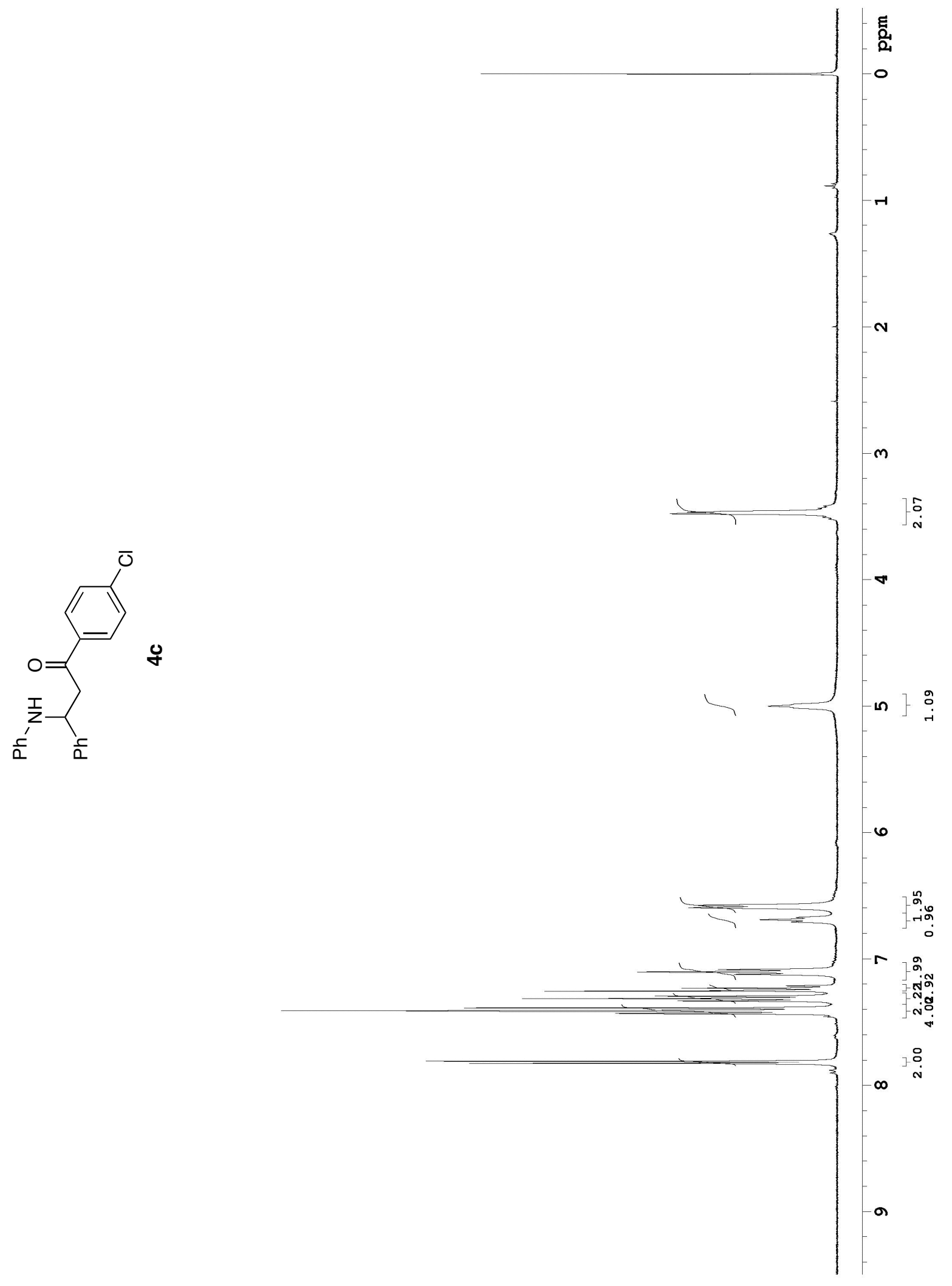



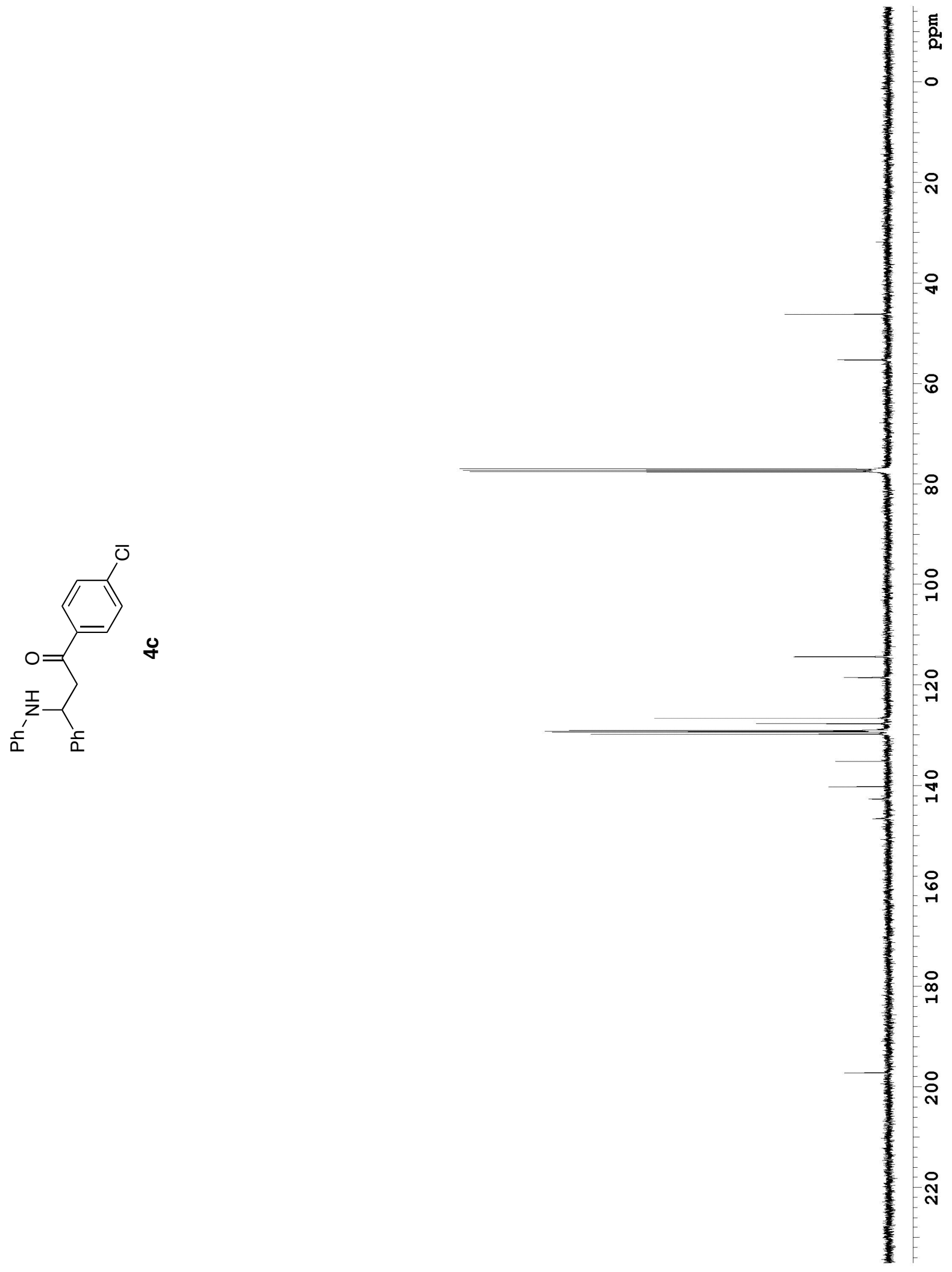

S-15 


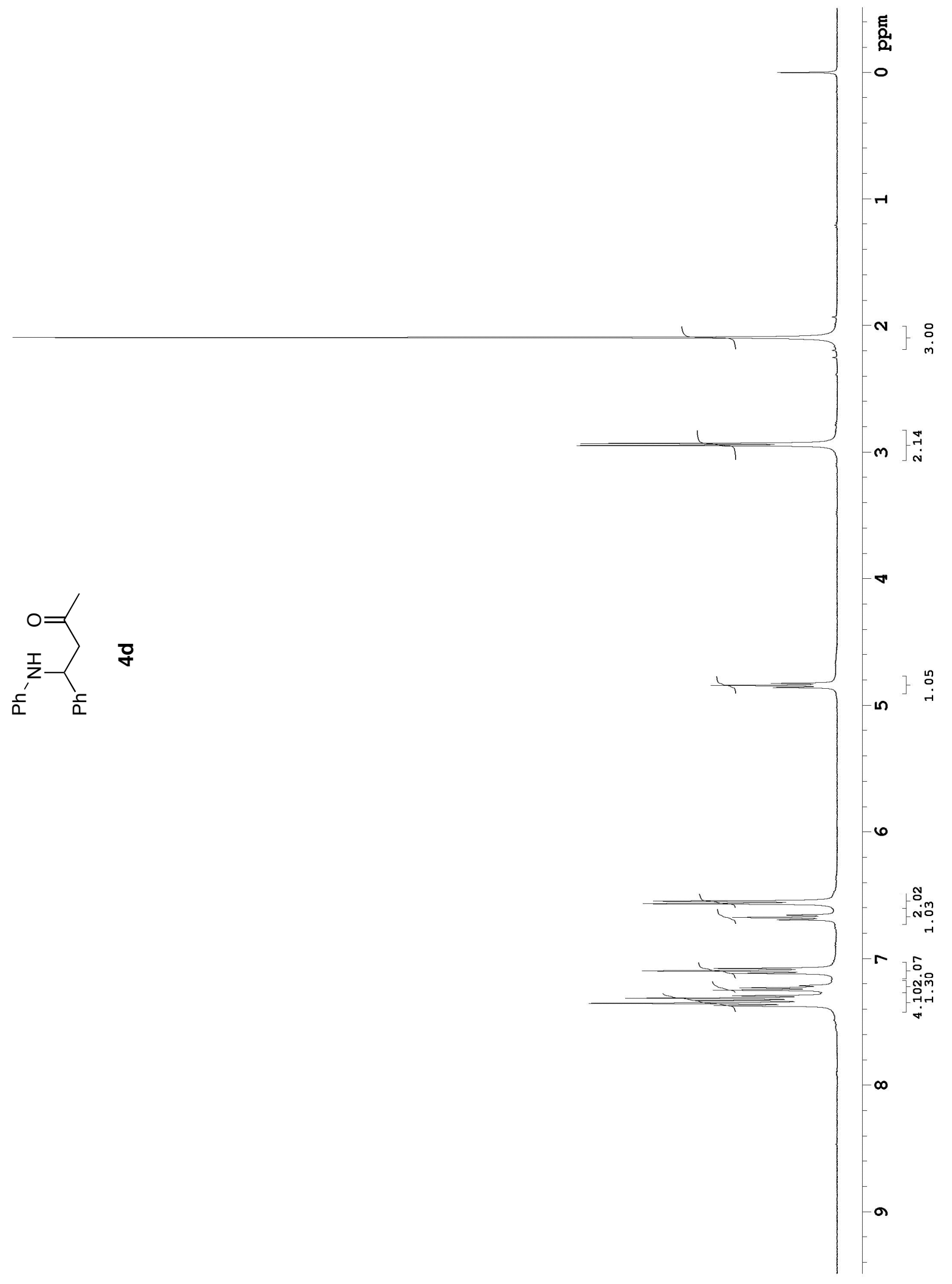

S-16 

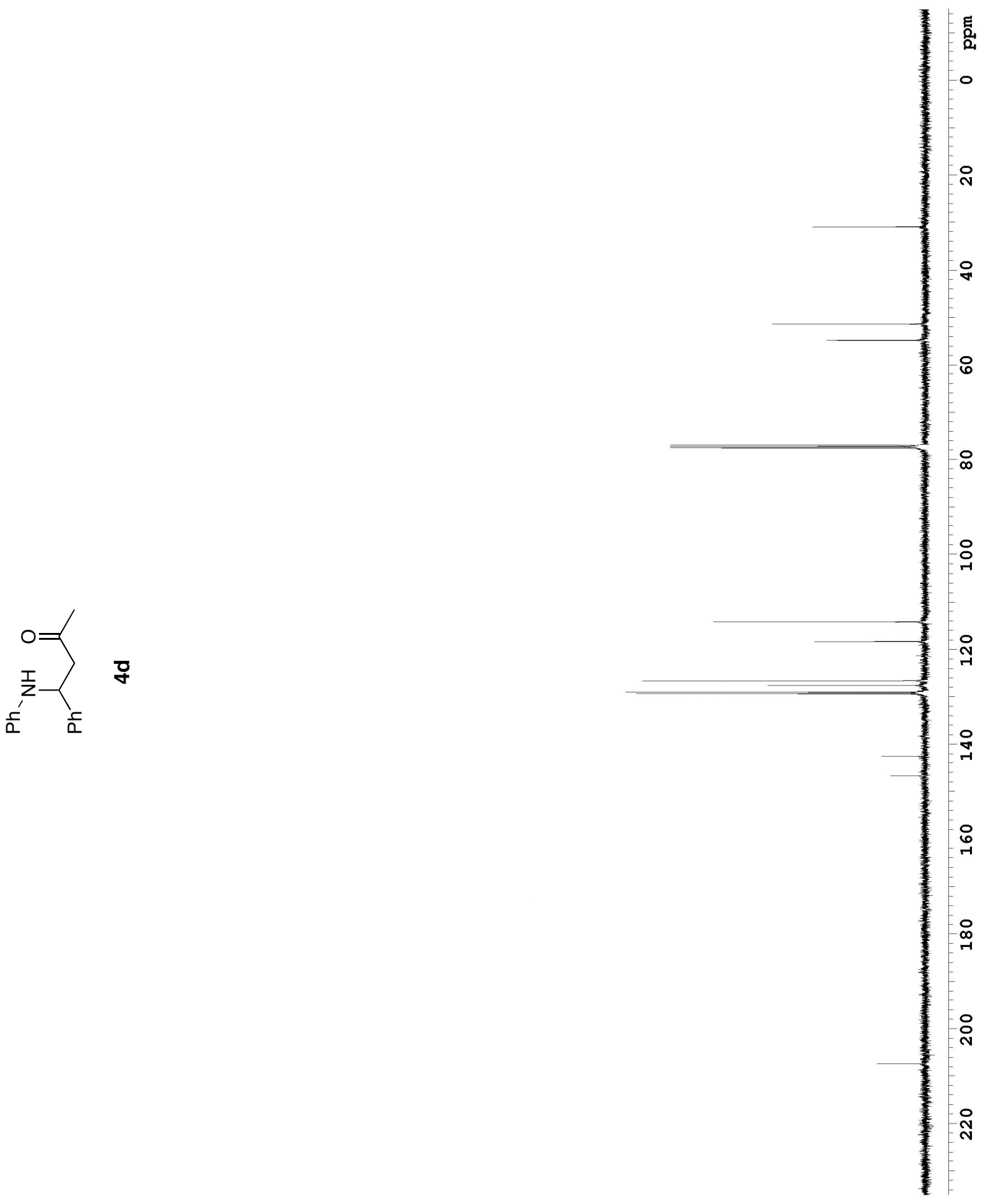

S-17 


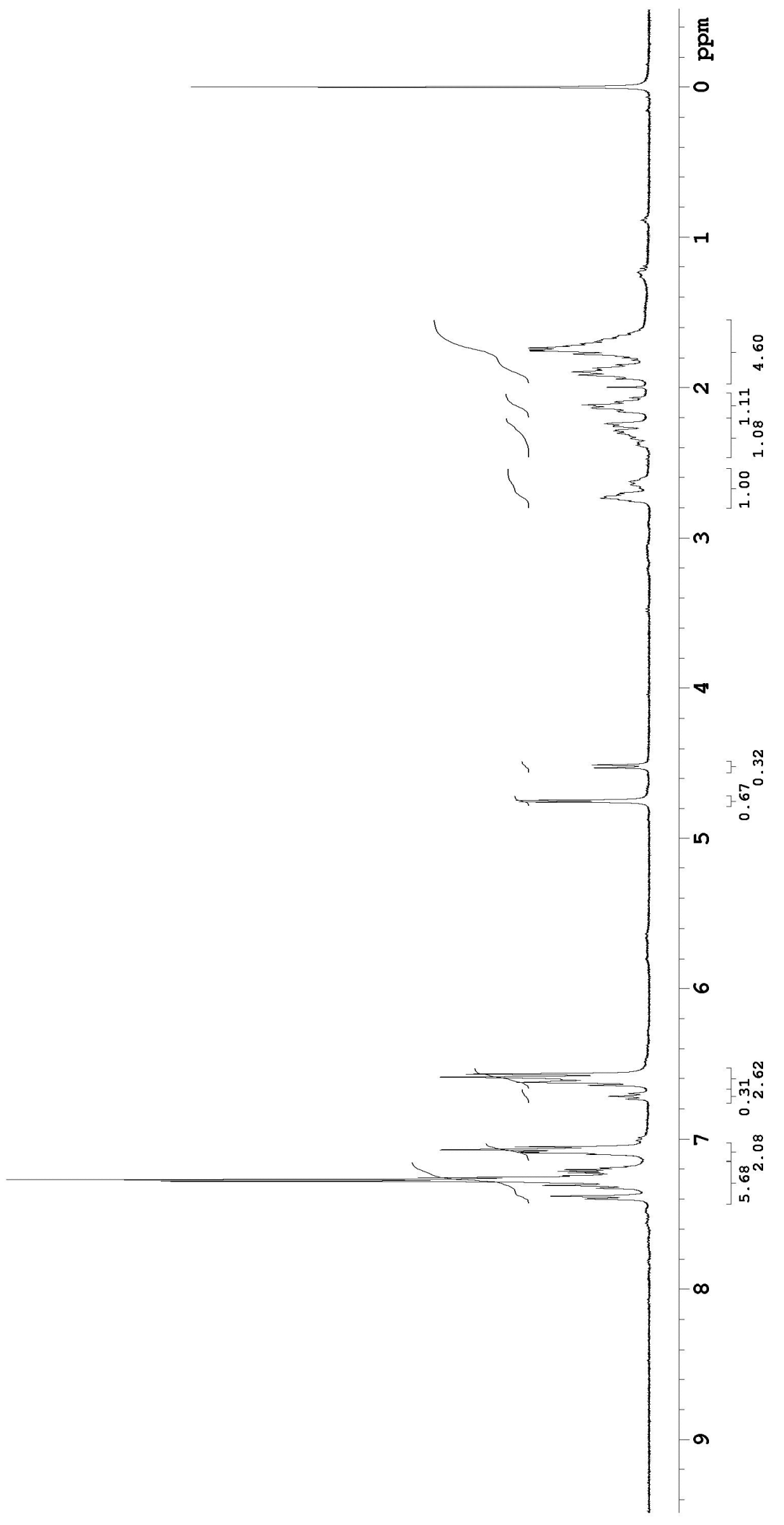

S-18 


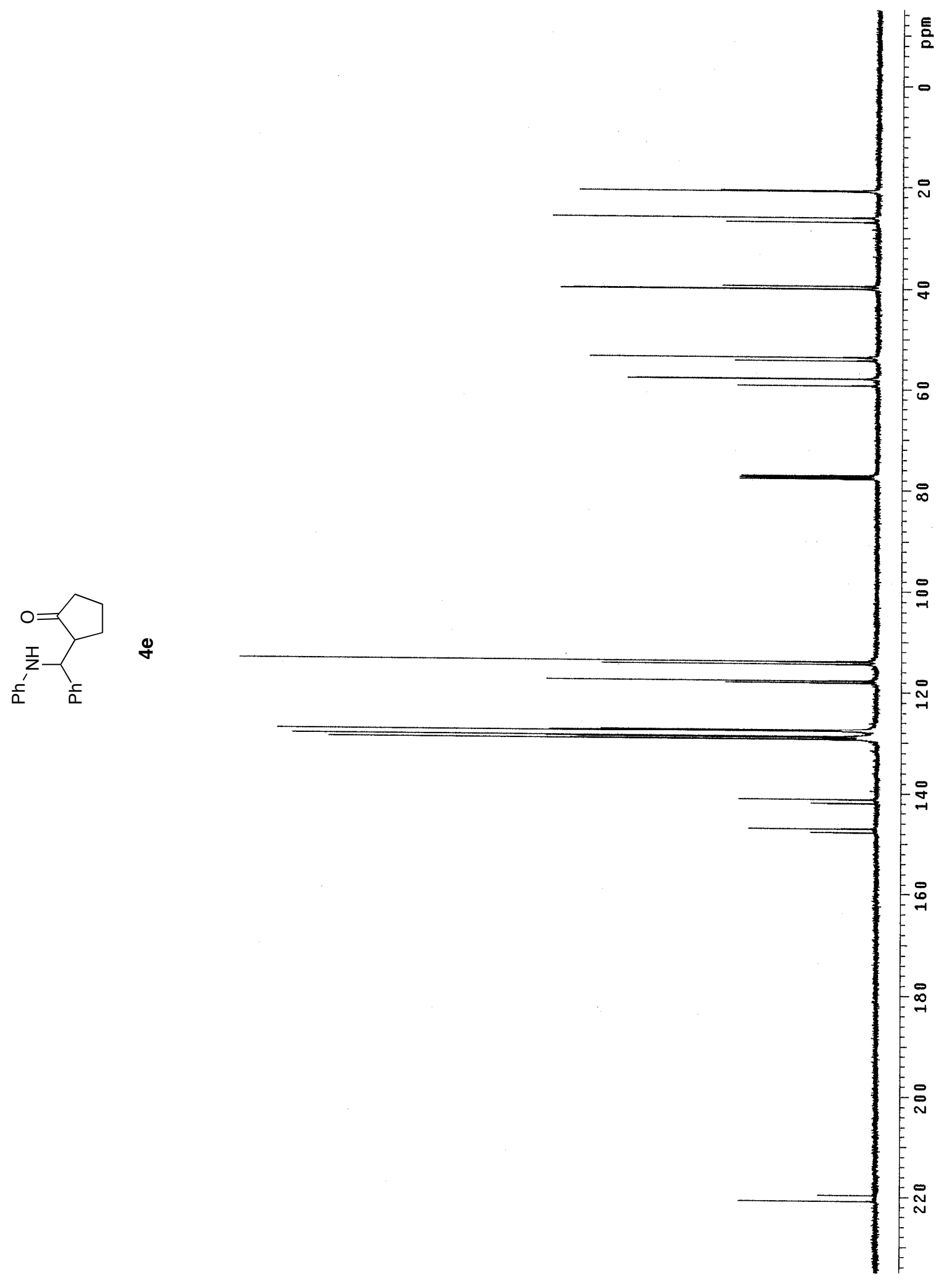

S-19 


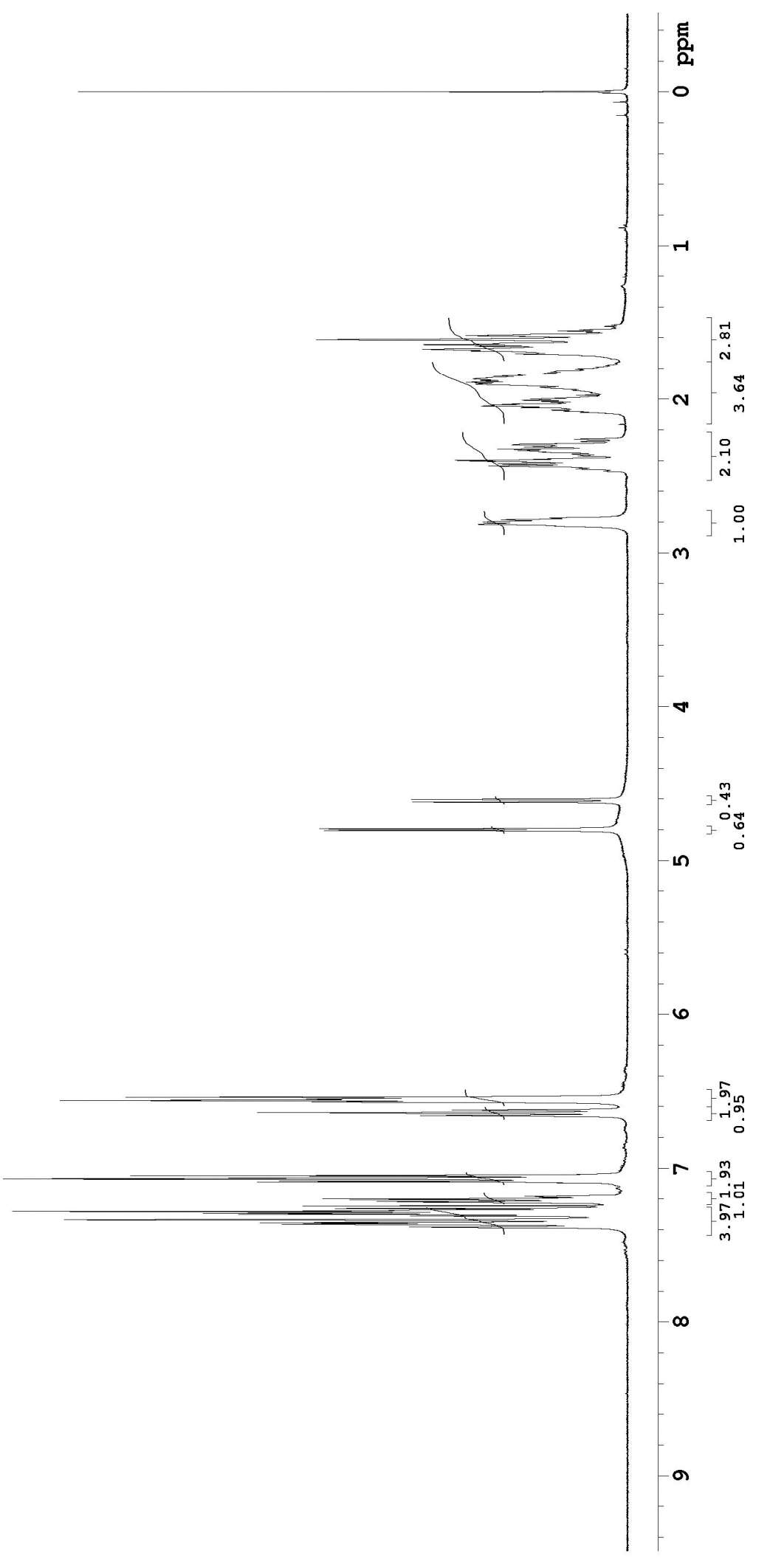




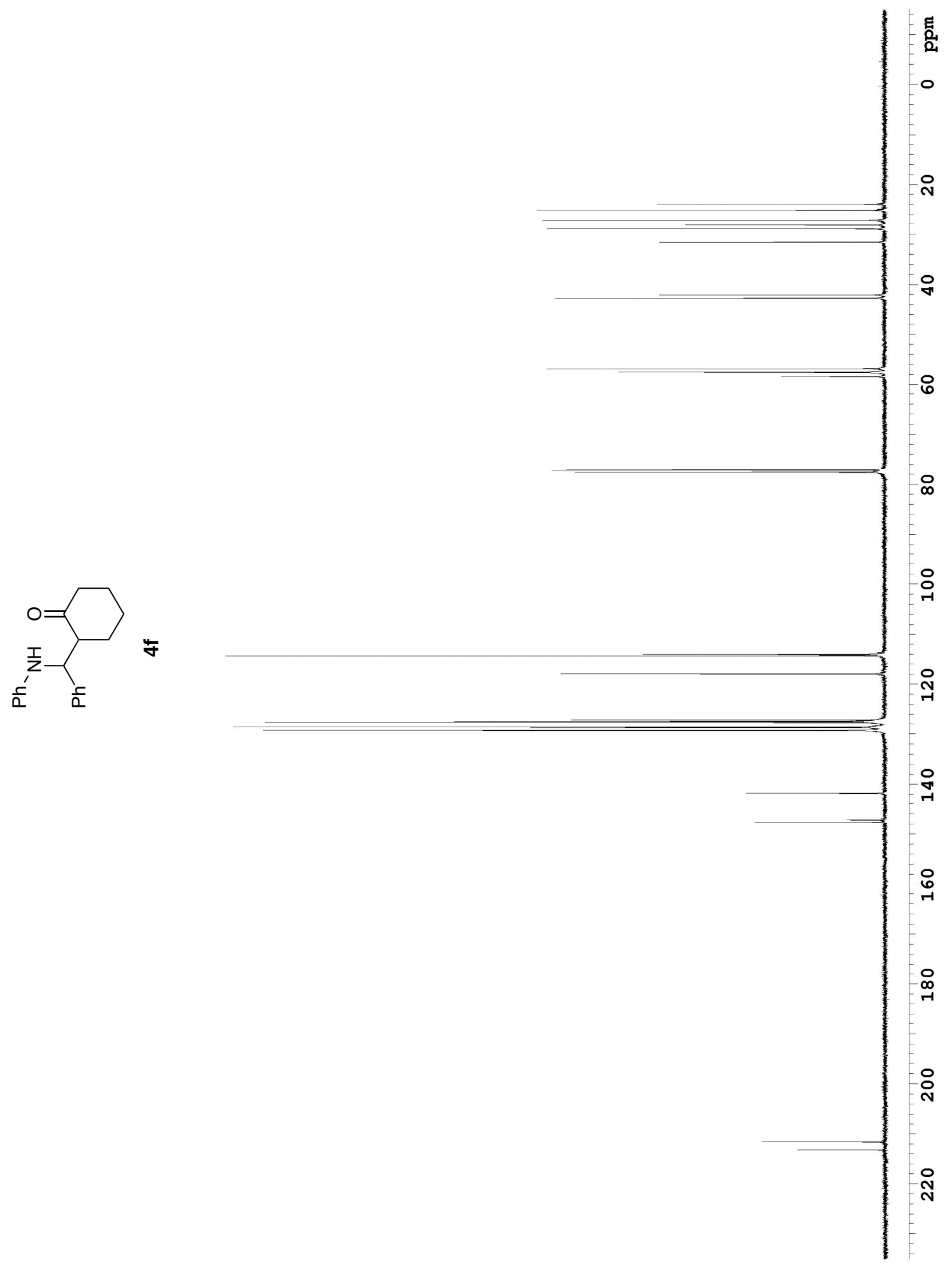




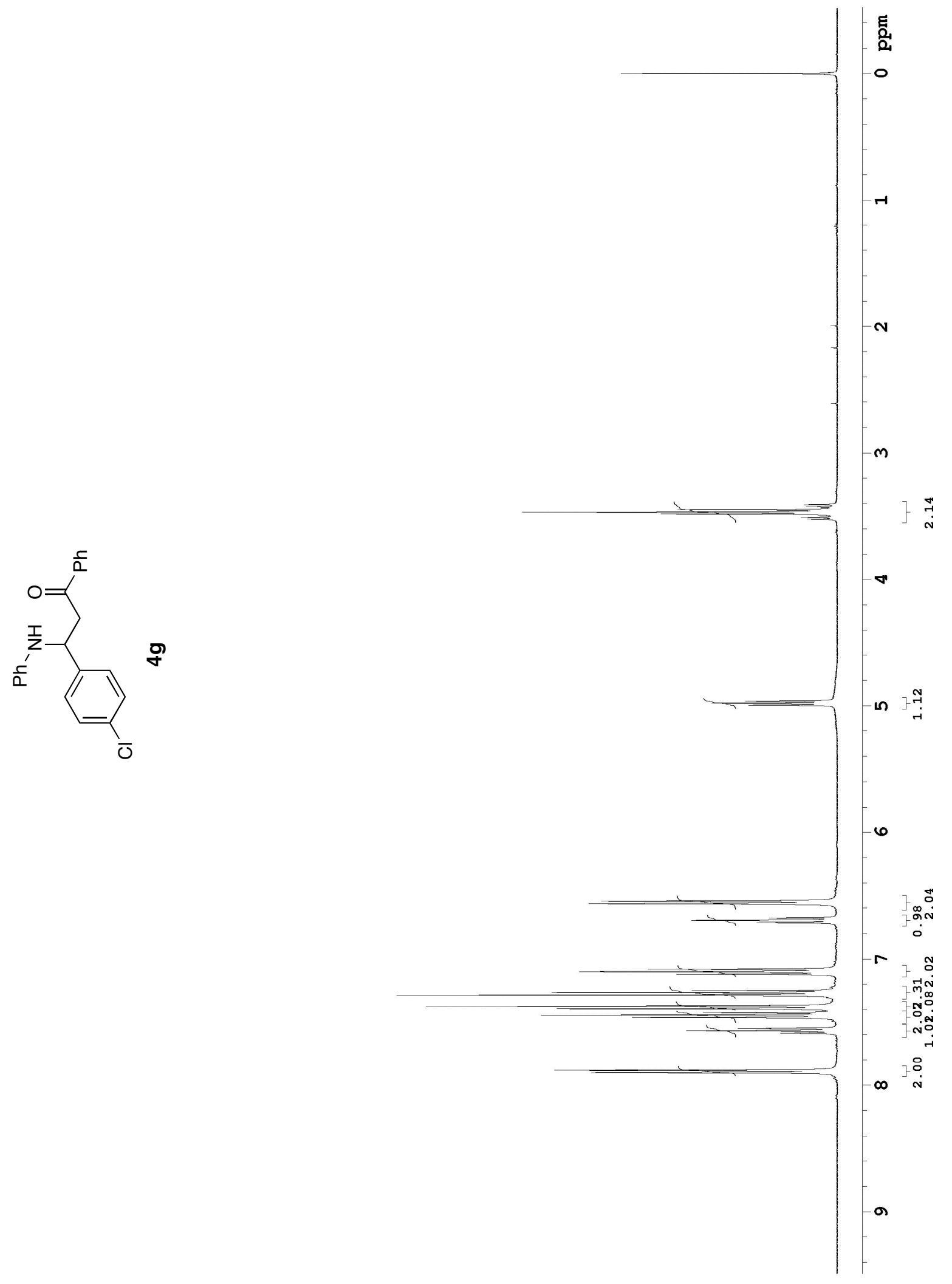




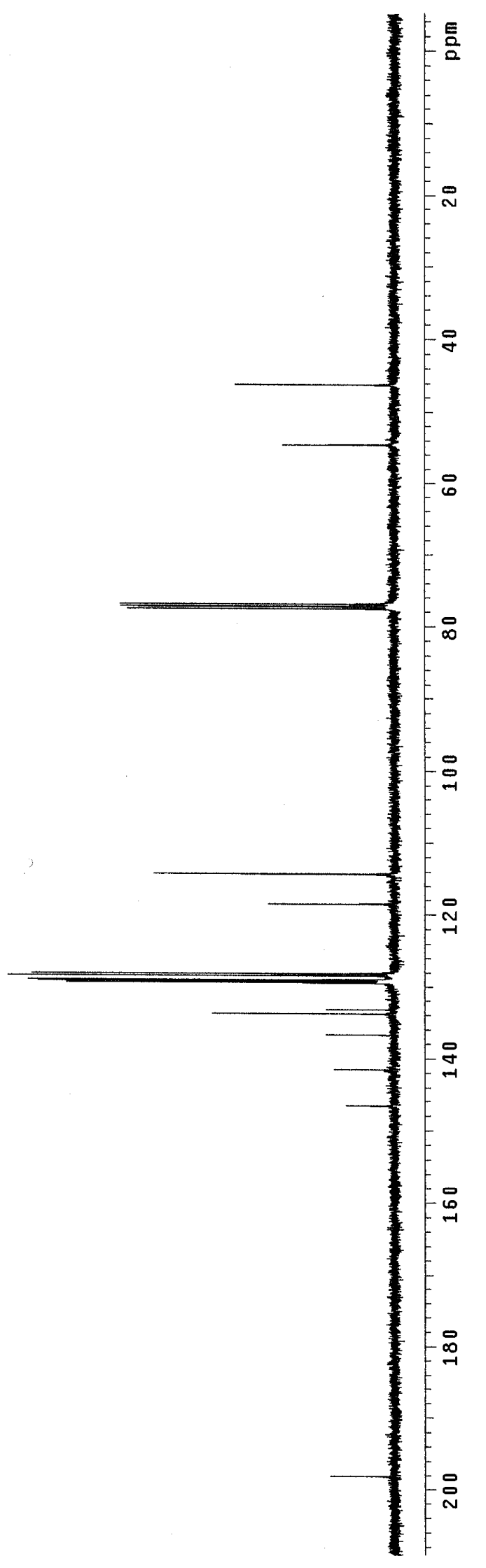

S-23 


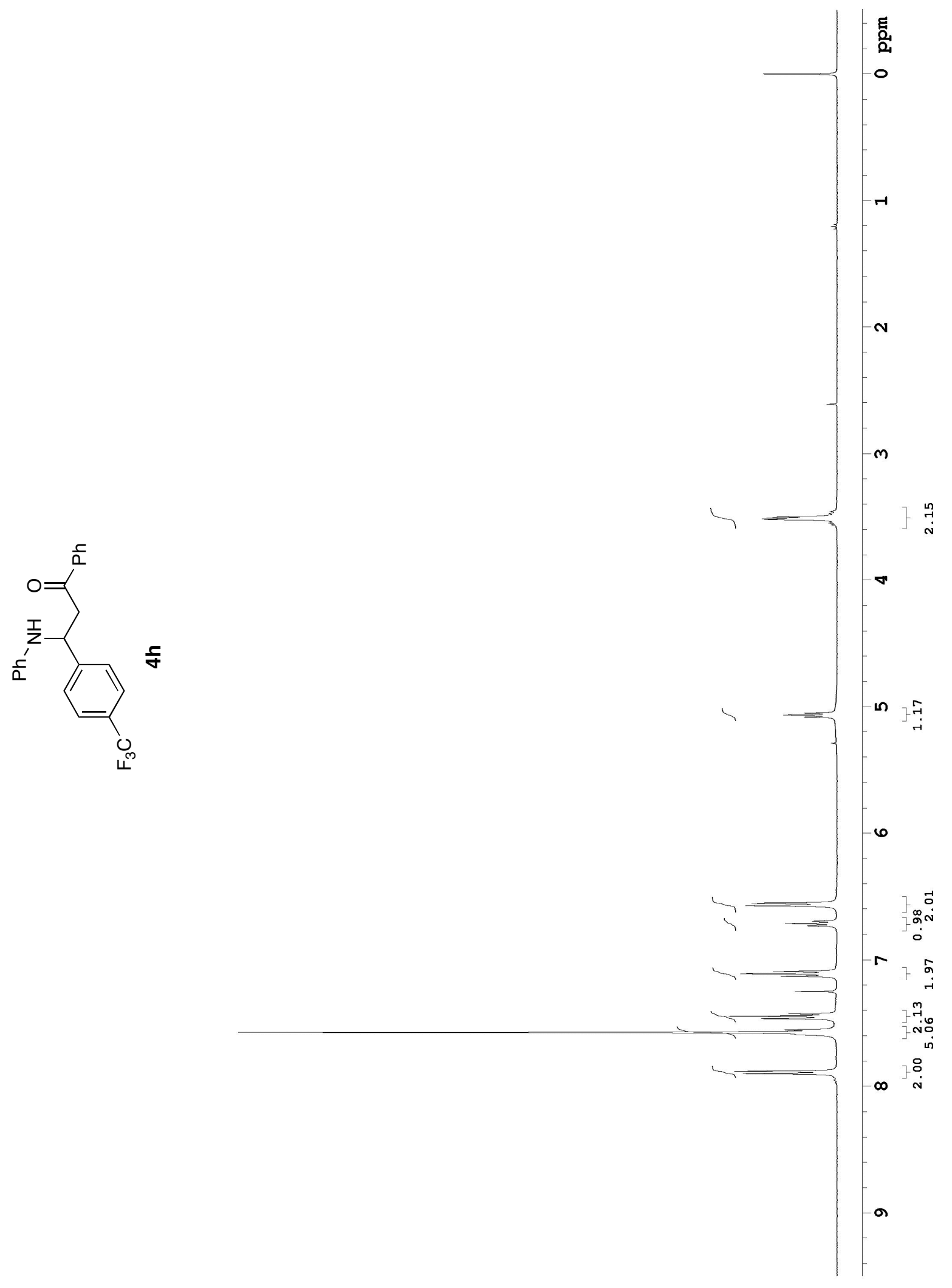




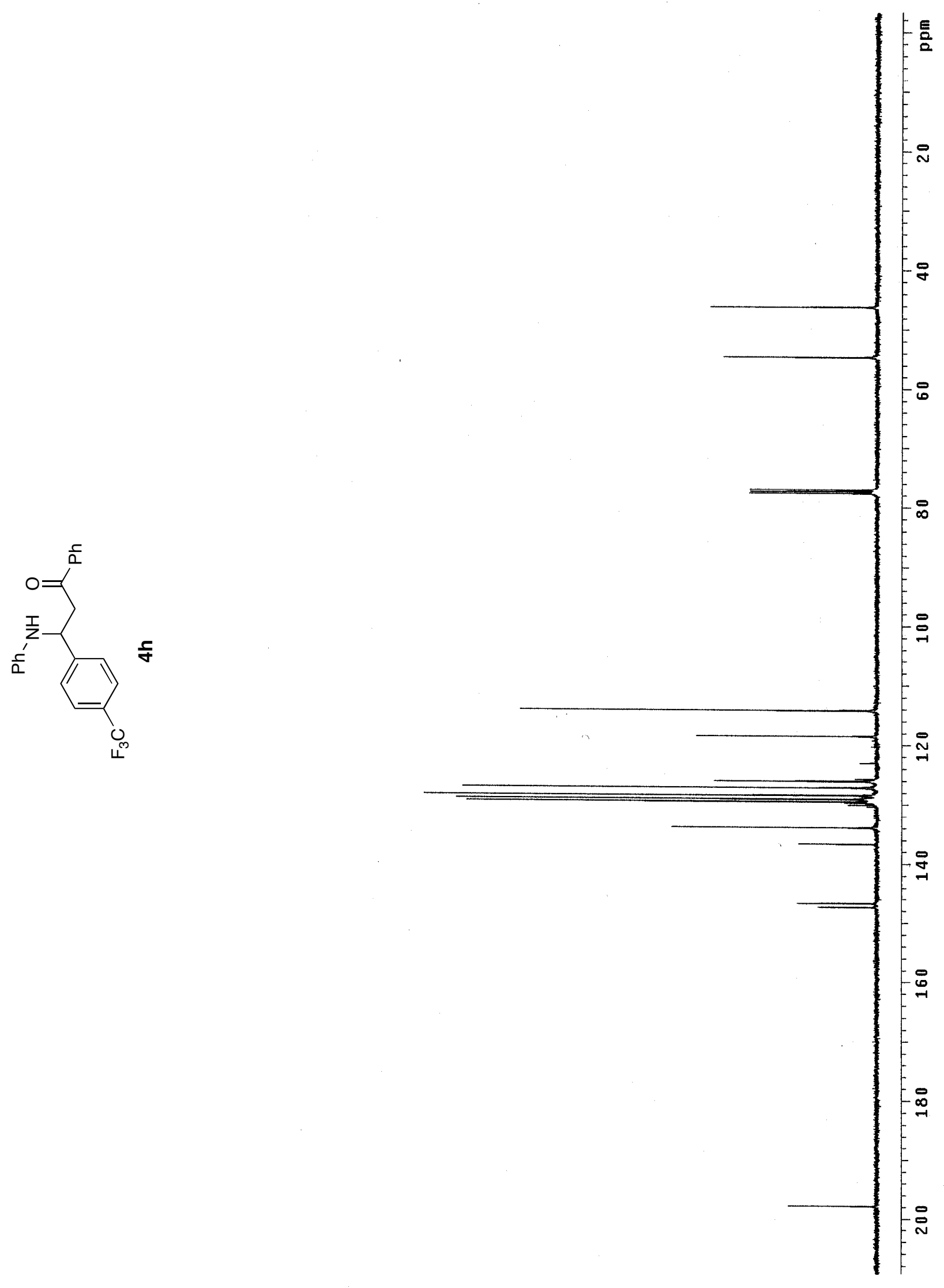




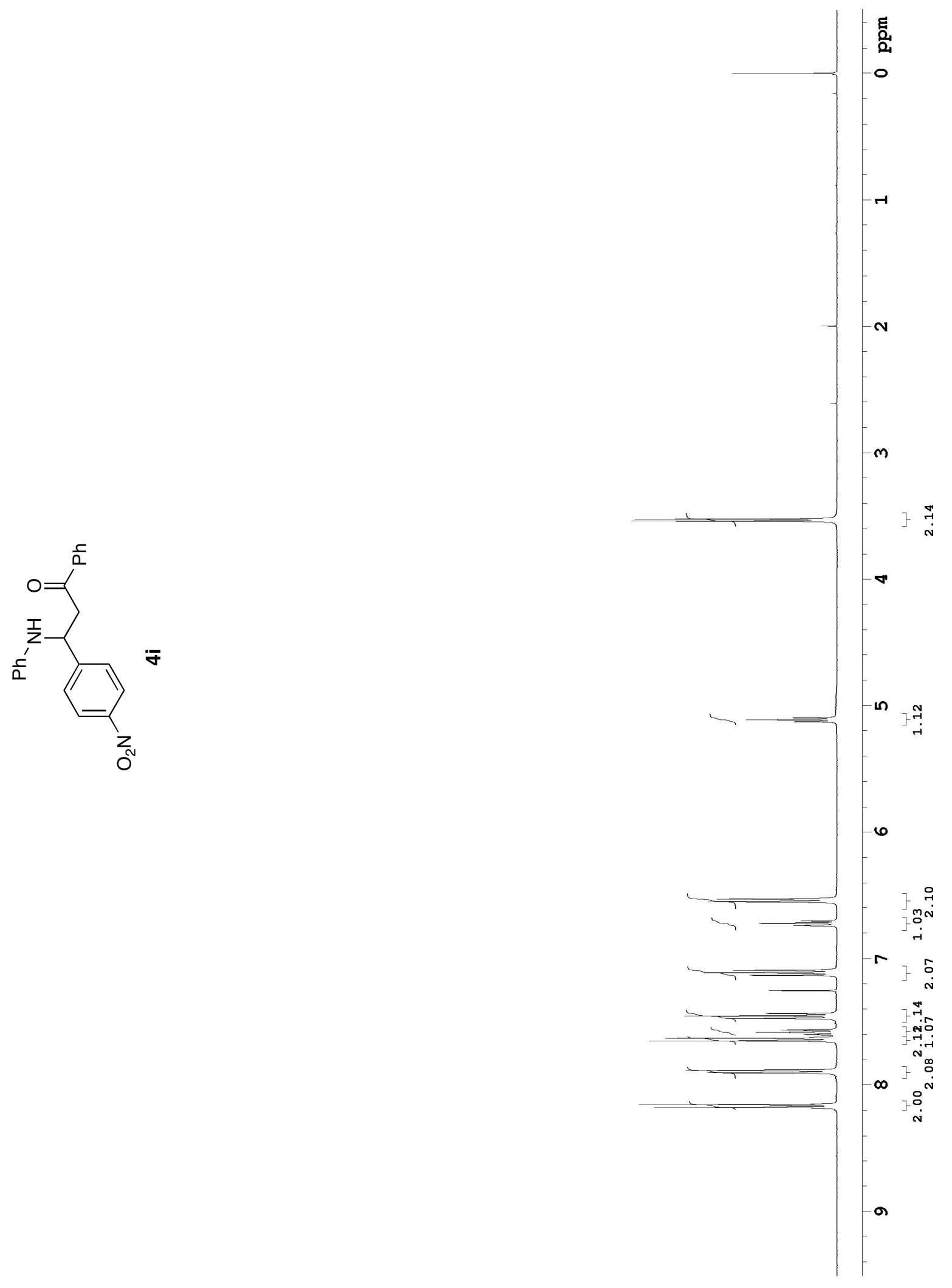




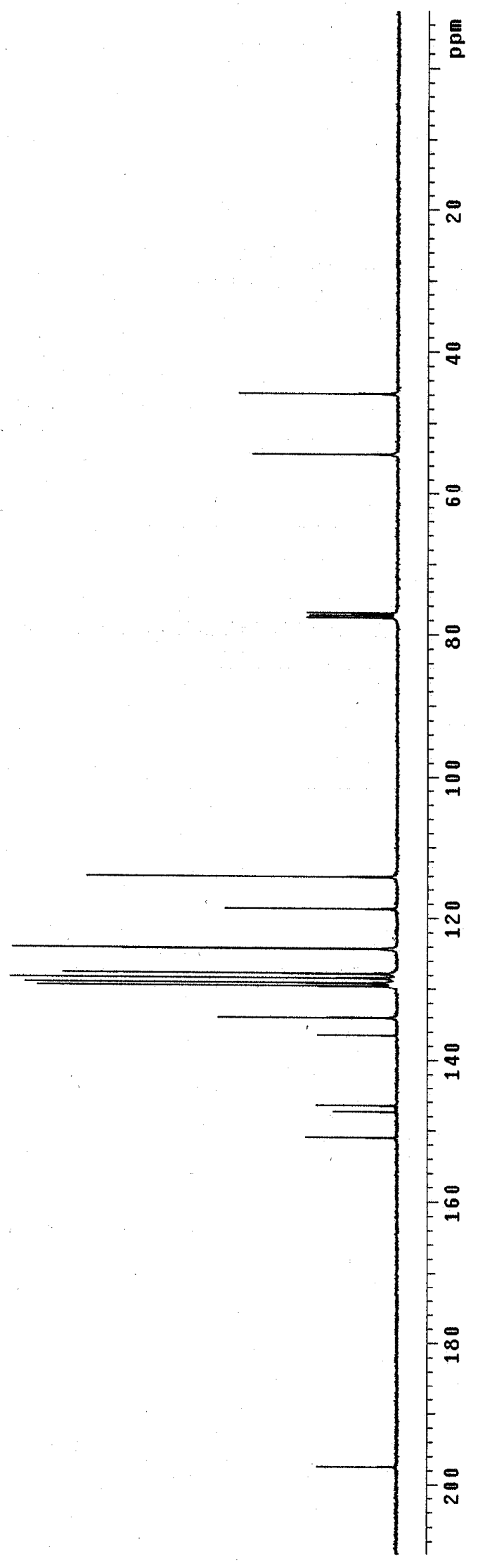




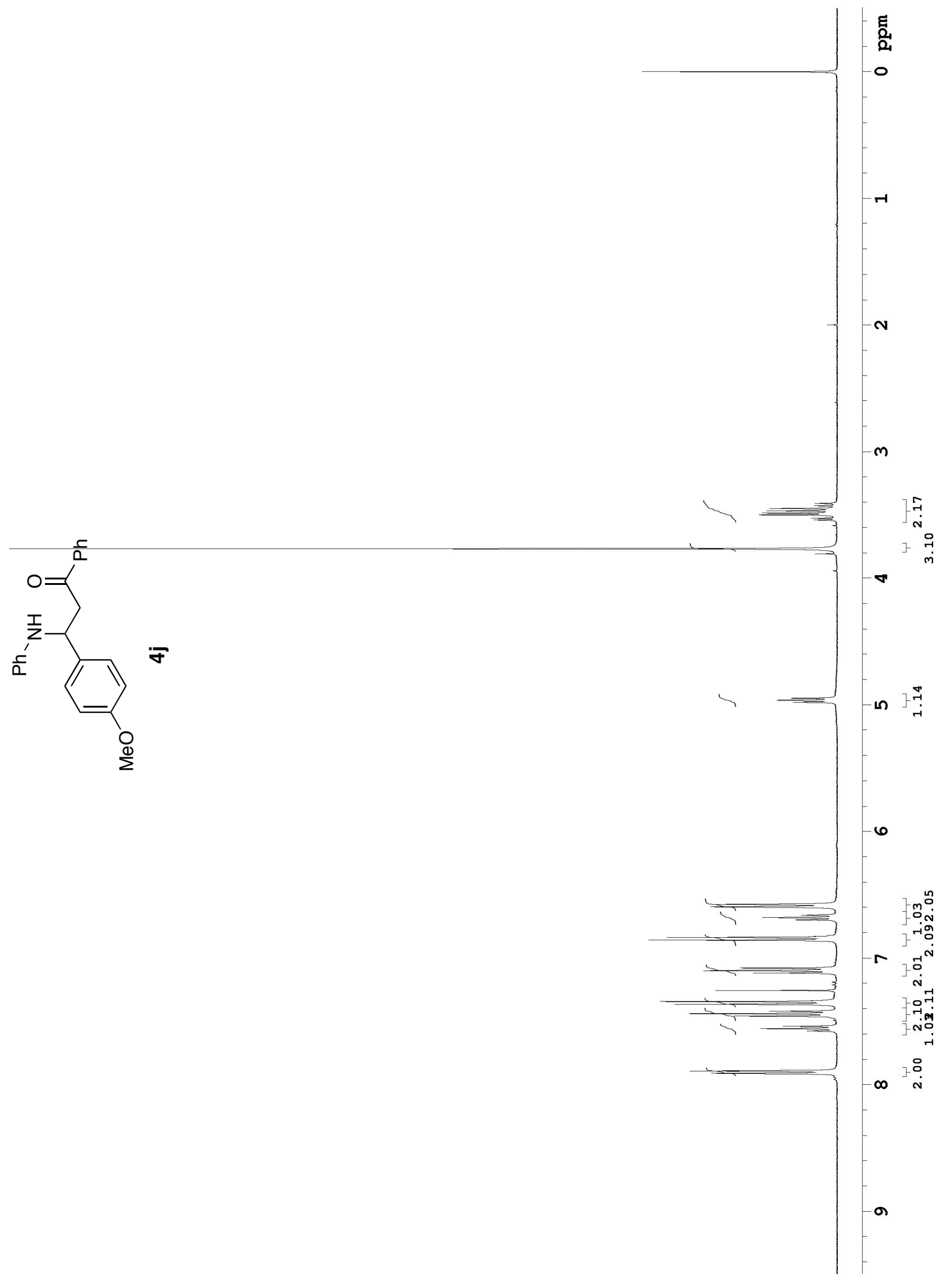




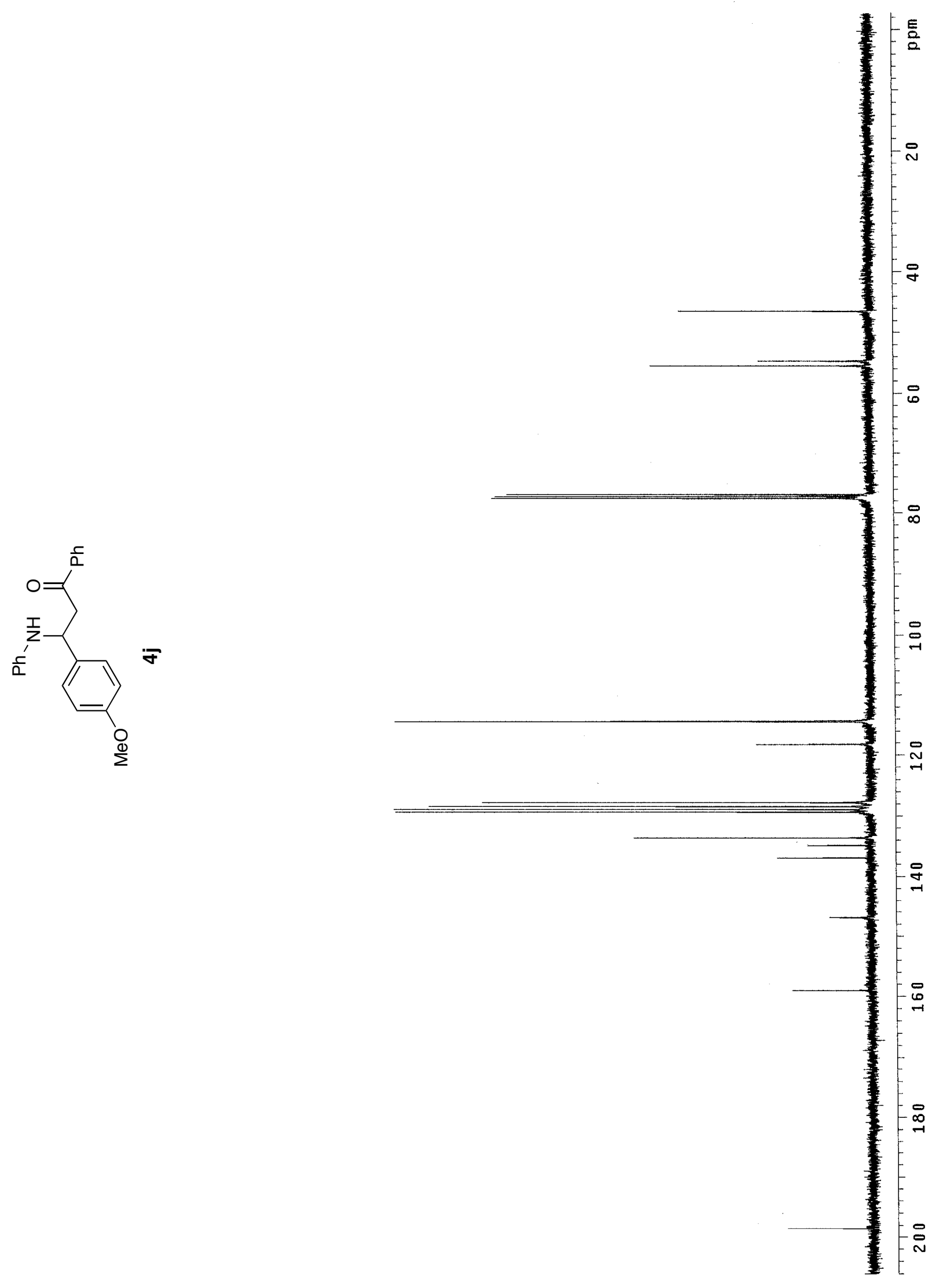




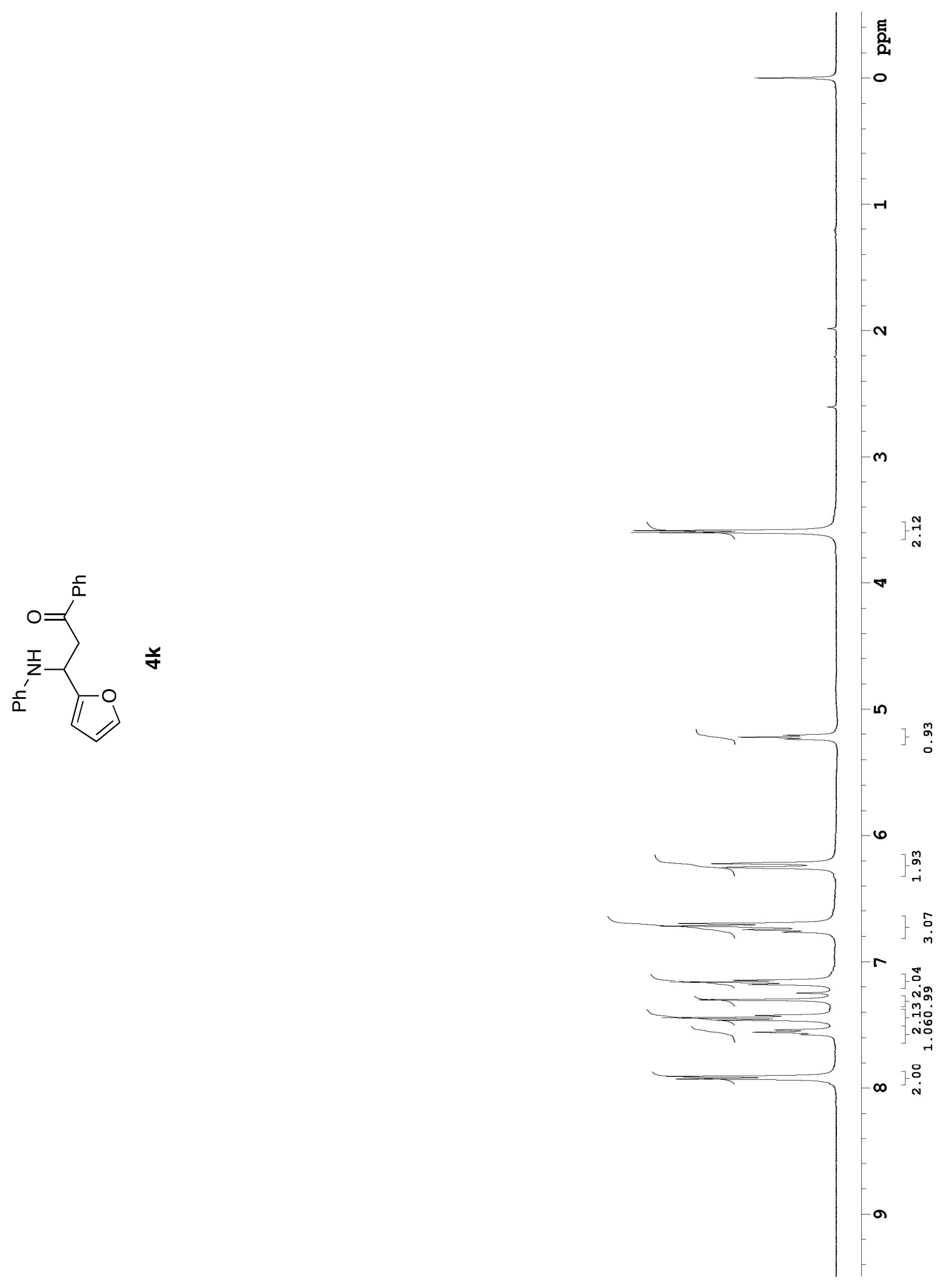




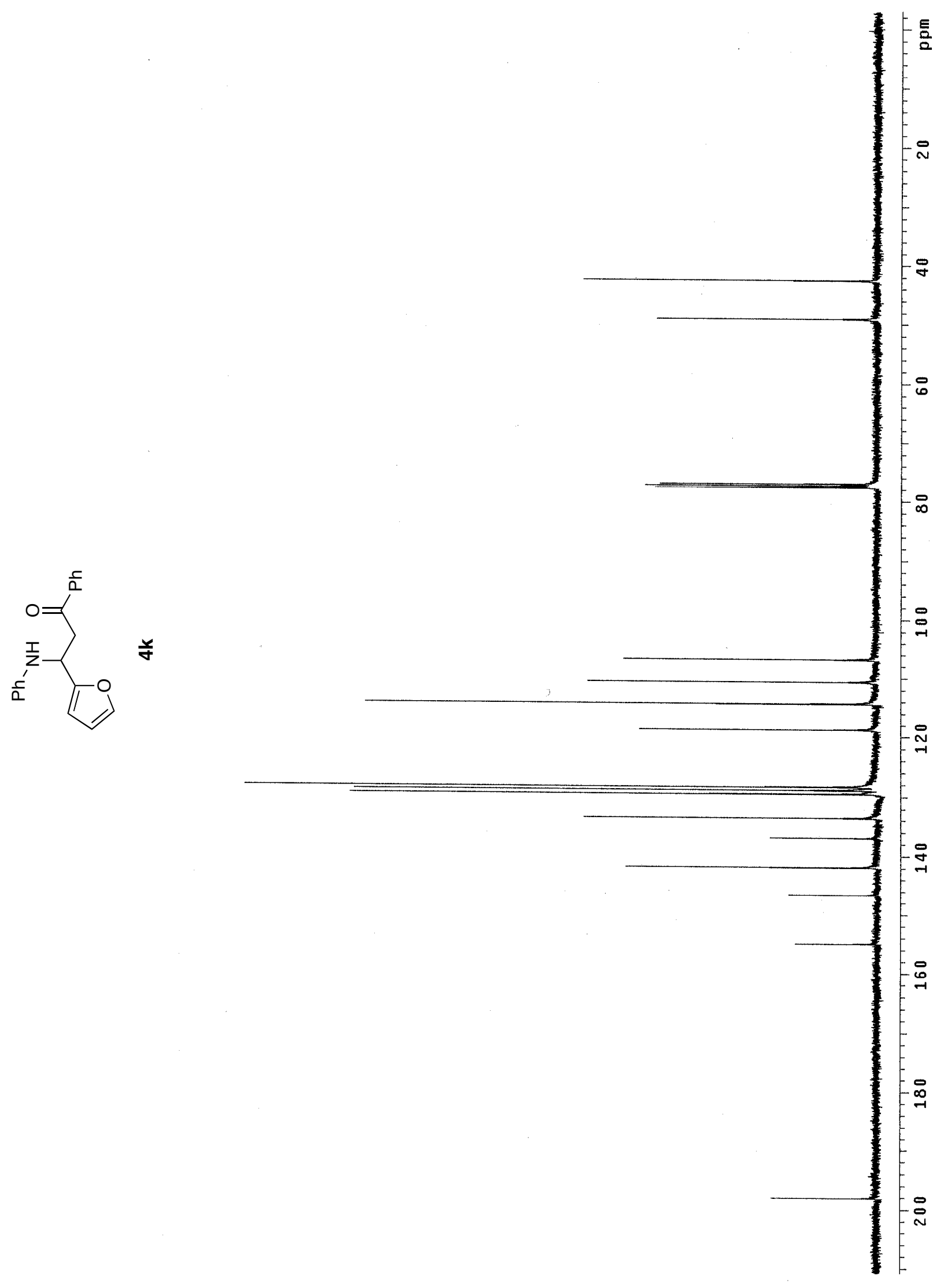

S-31 


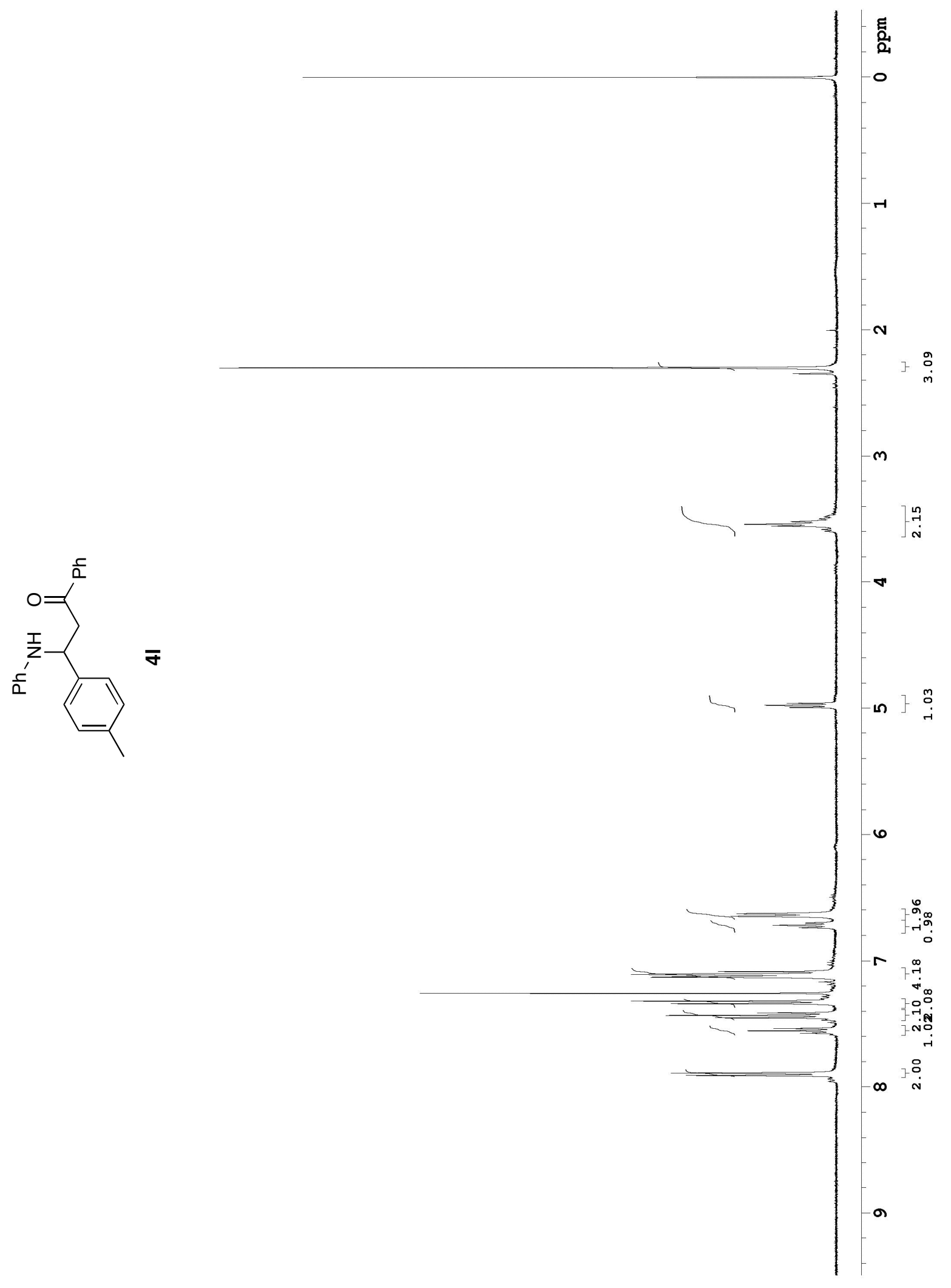




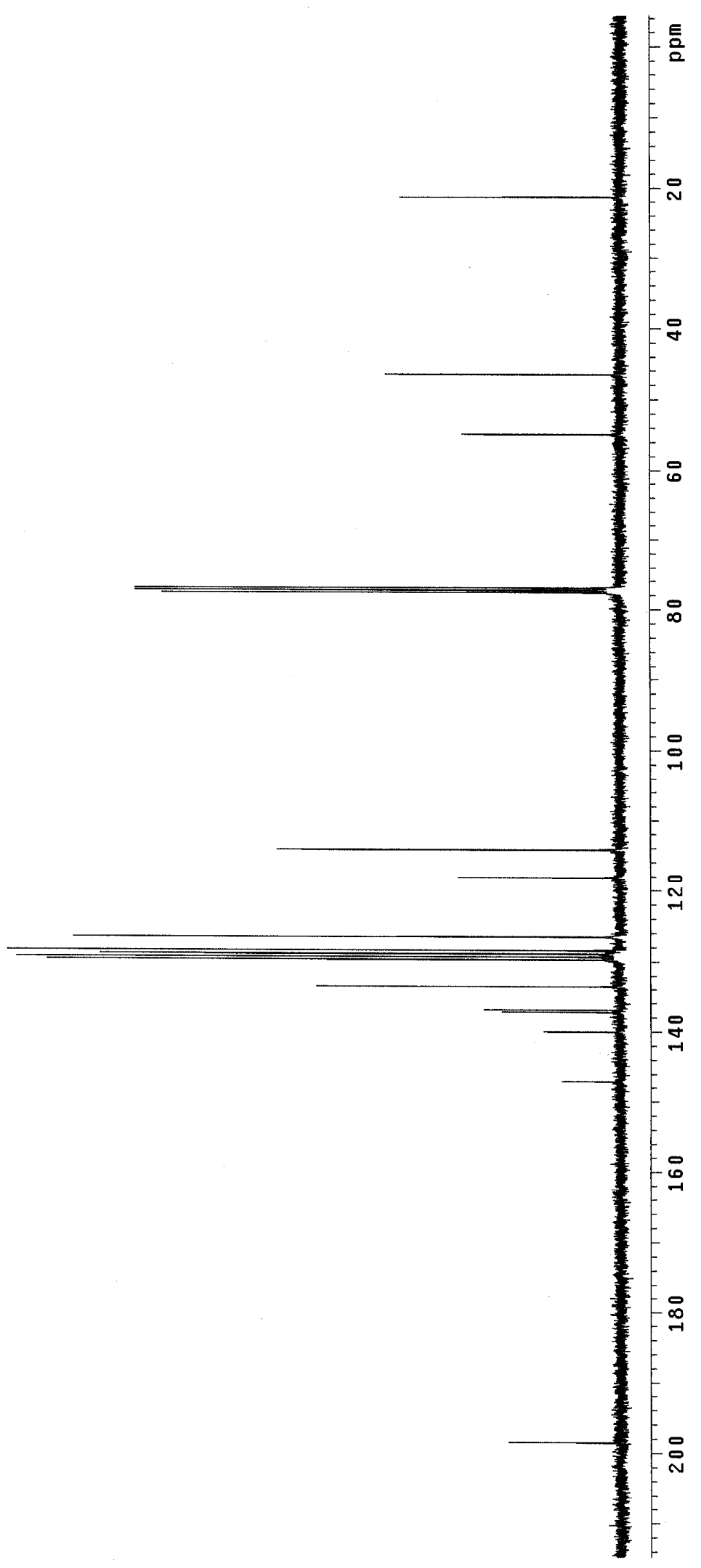

S-33 


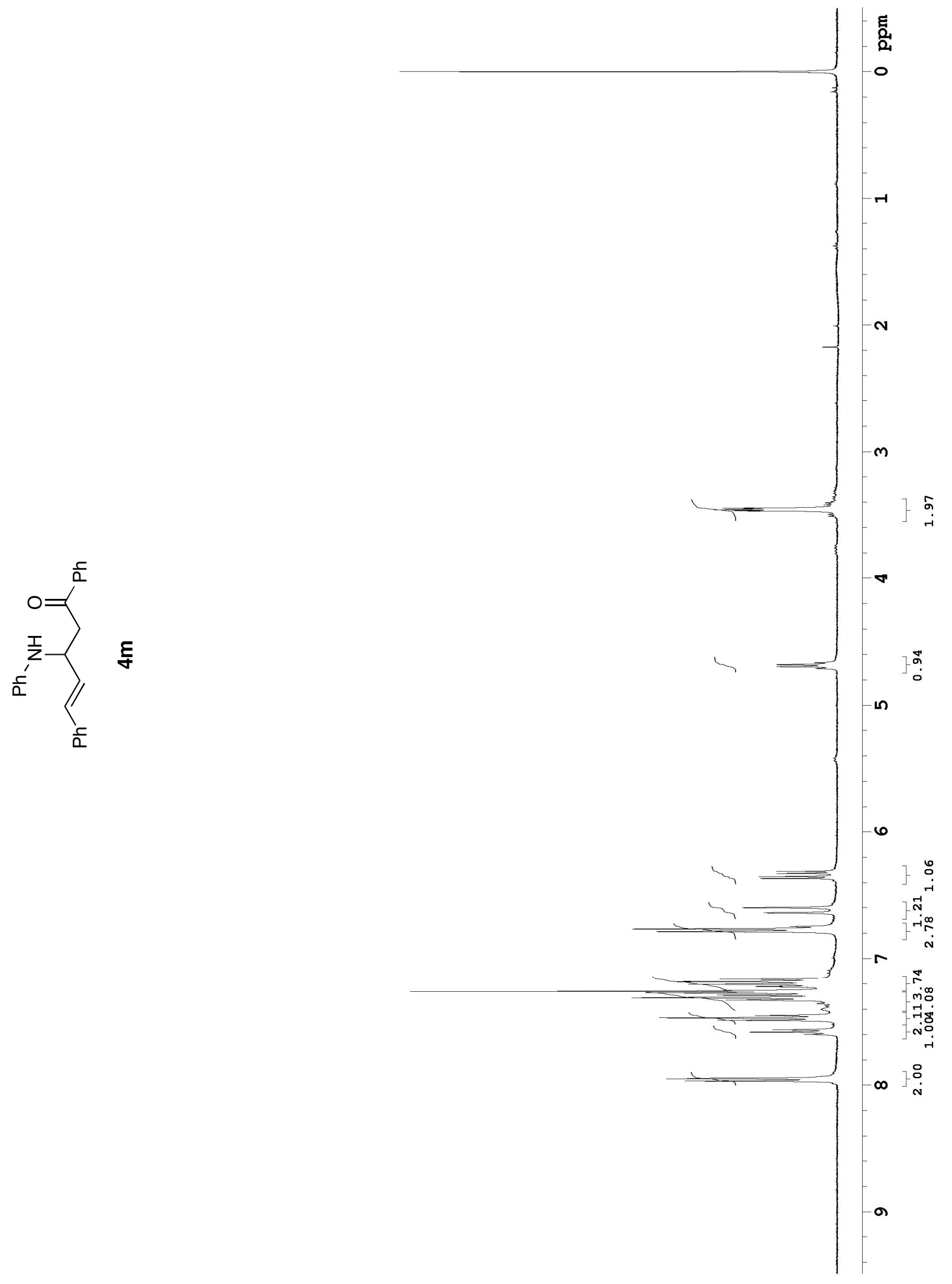




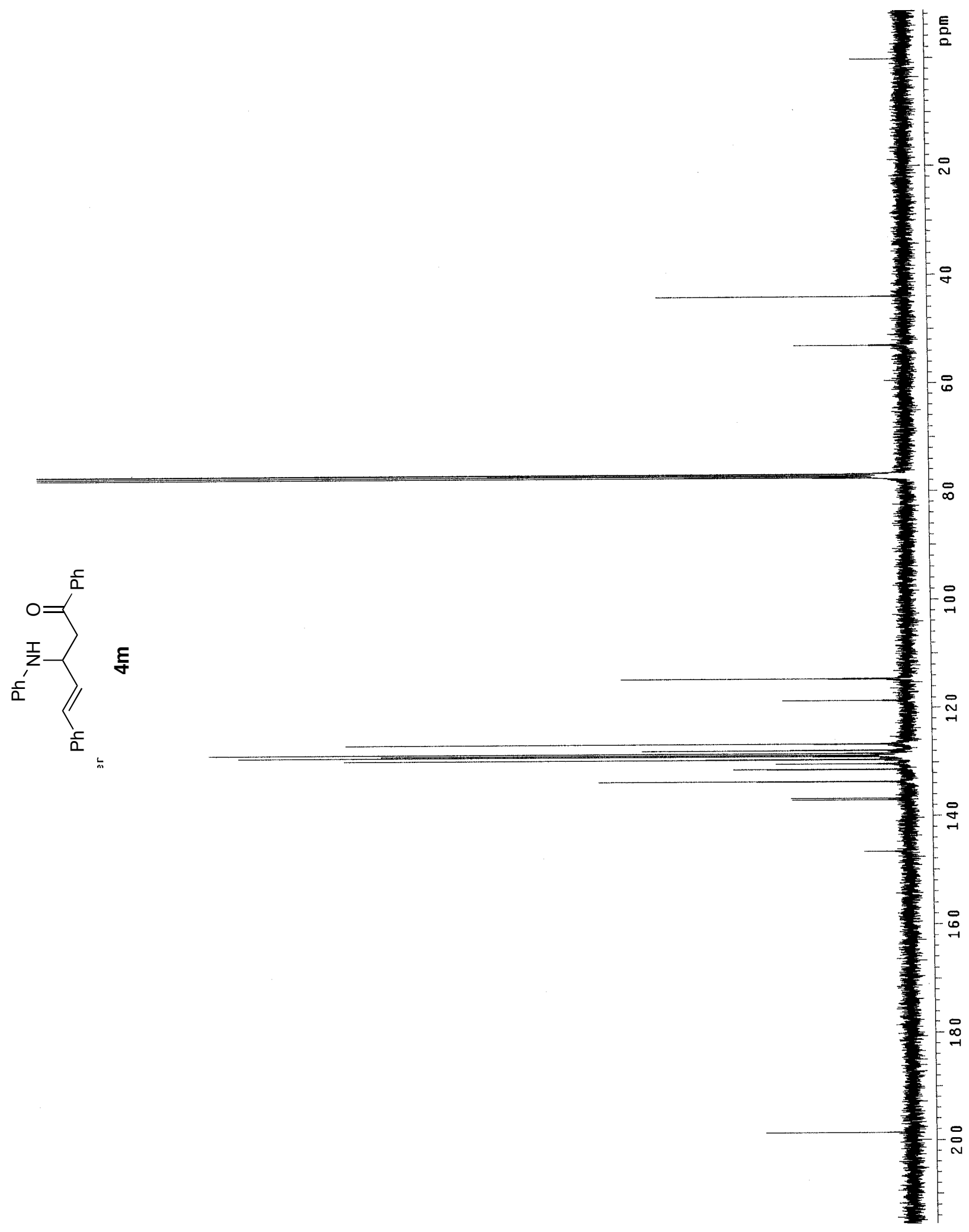




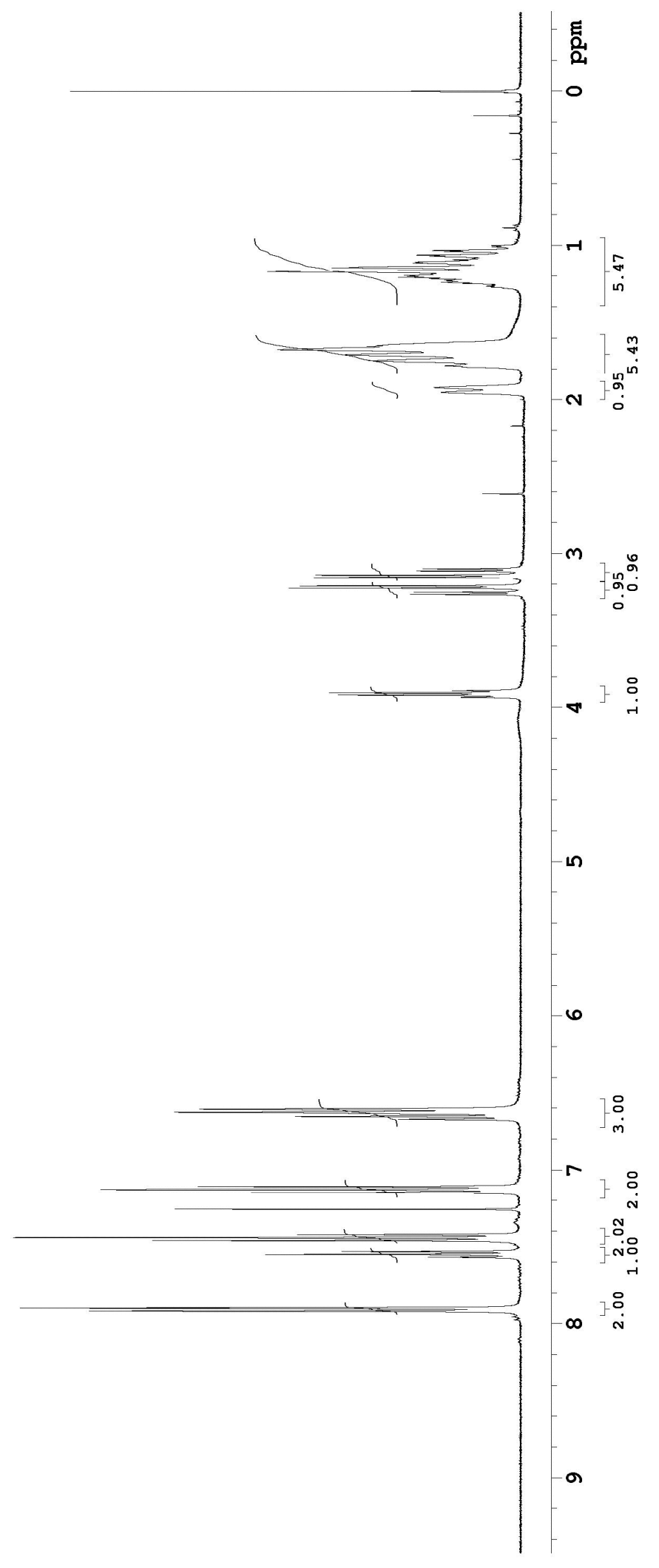

S-36 


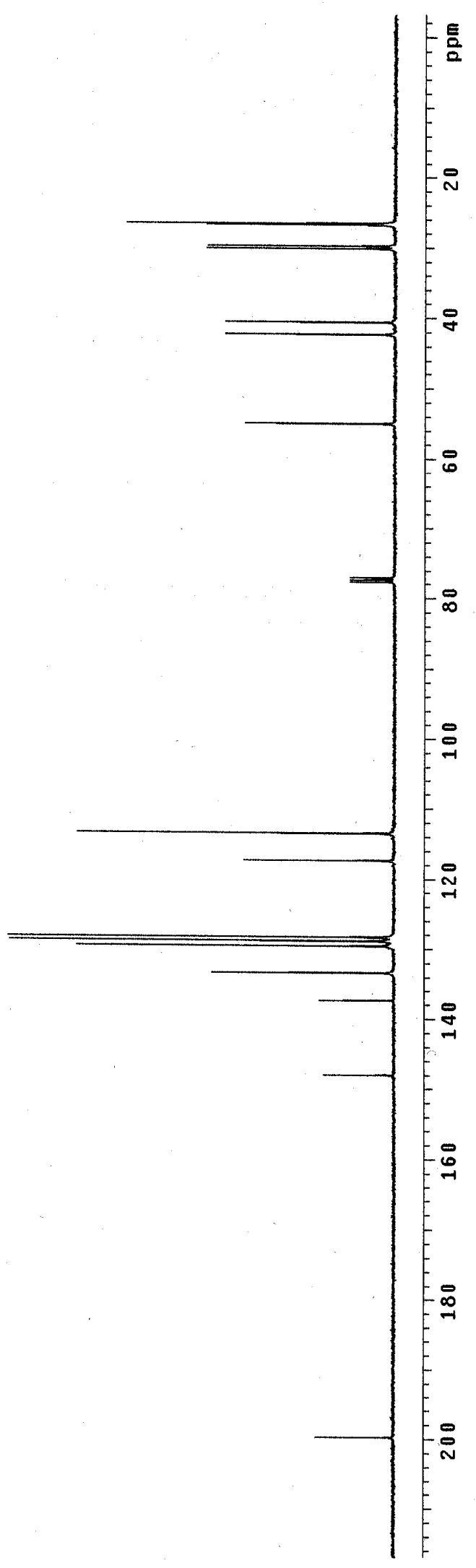




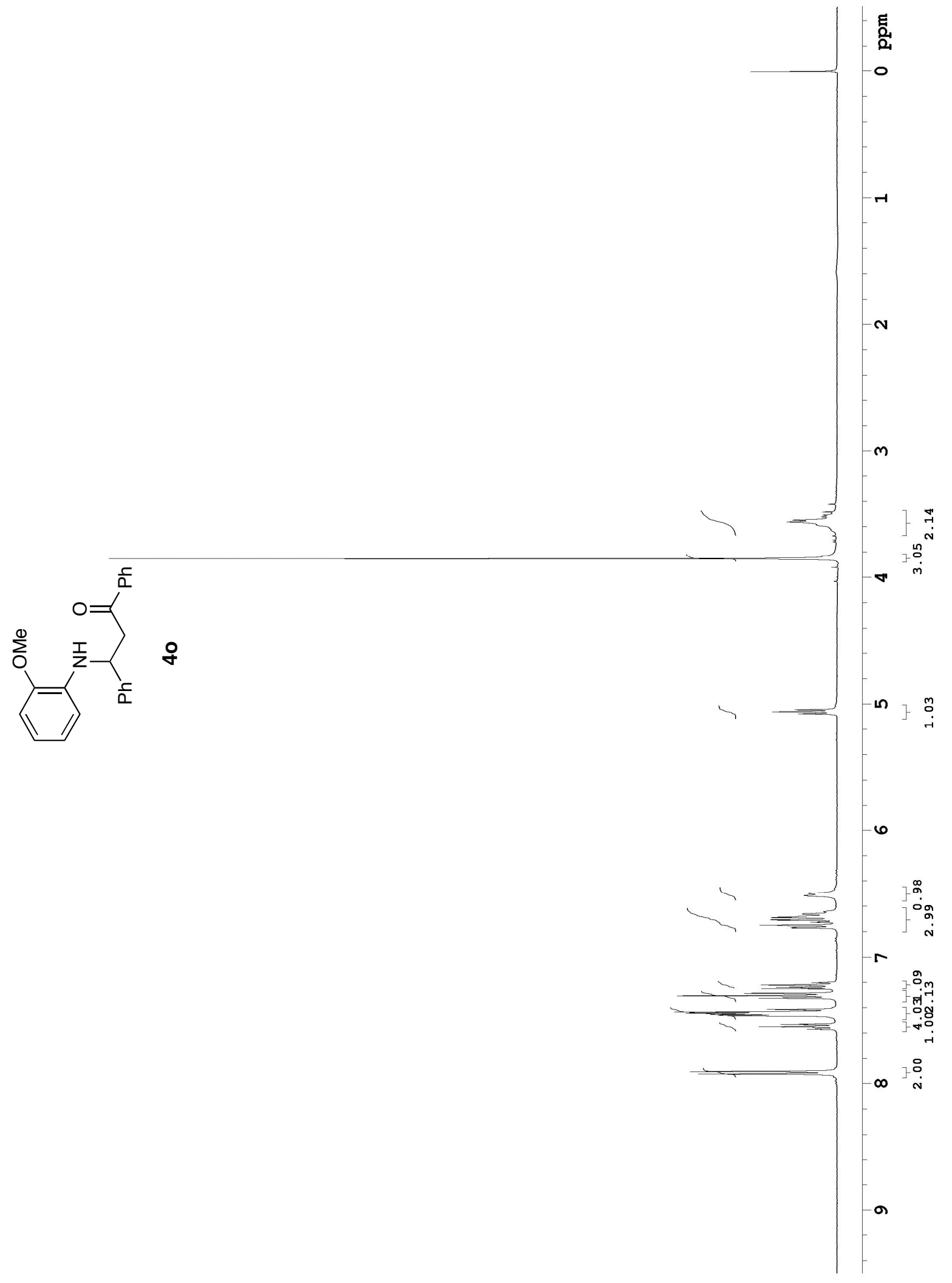

S-38 


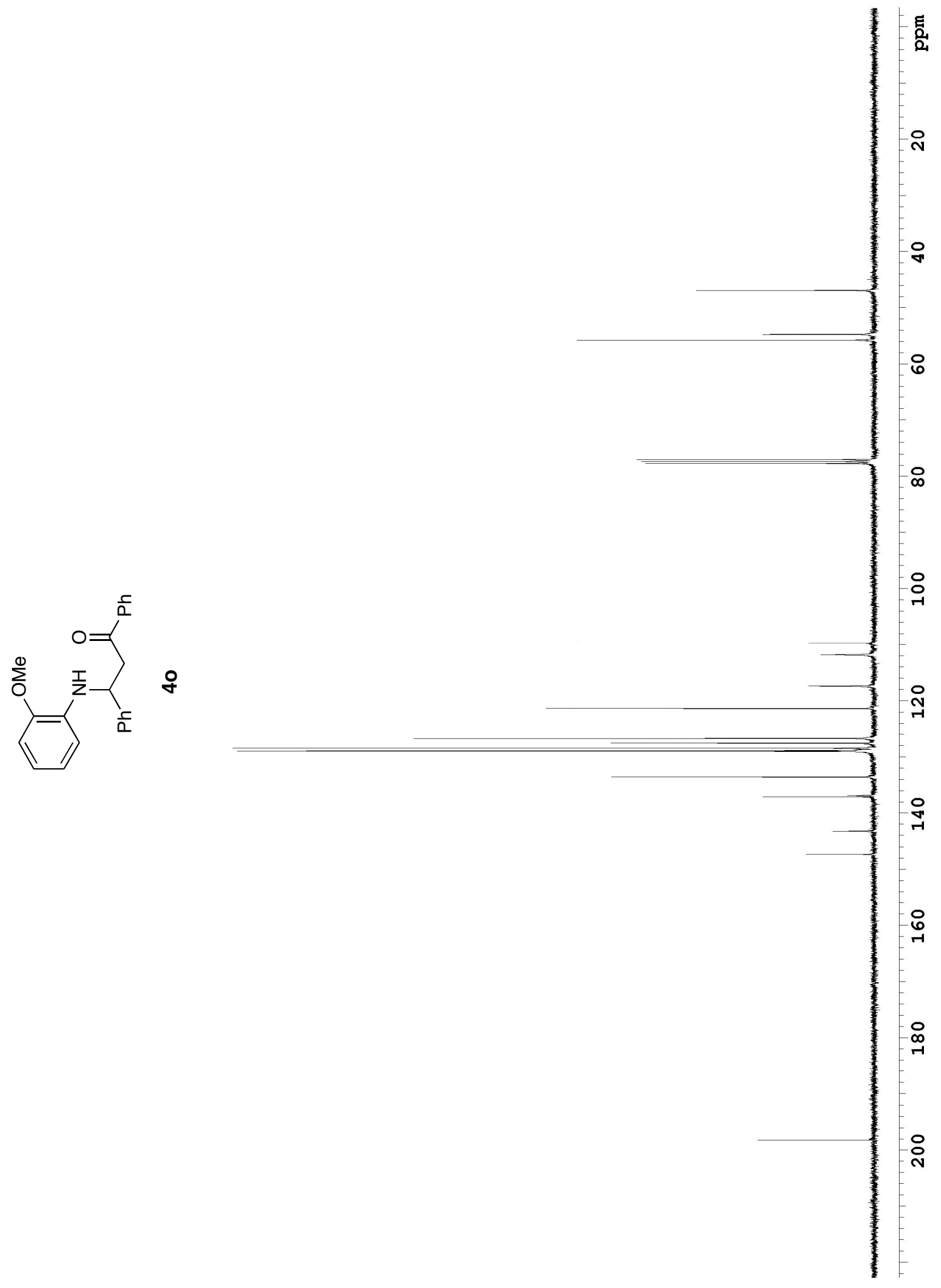




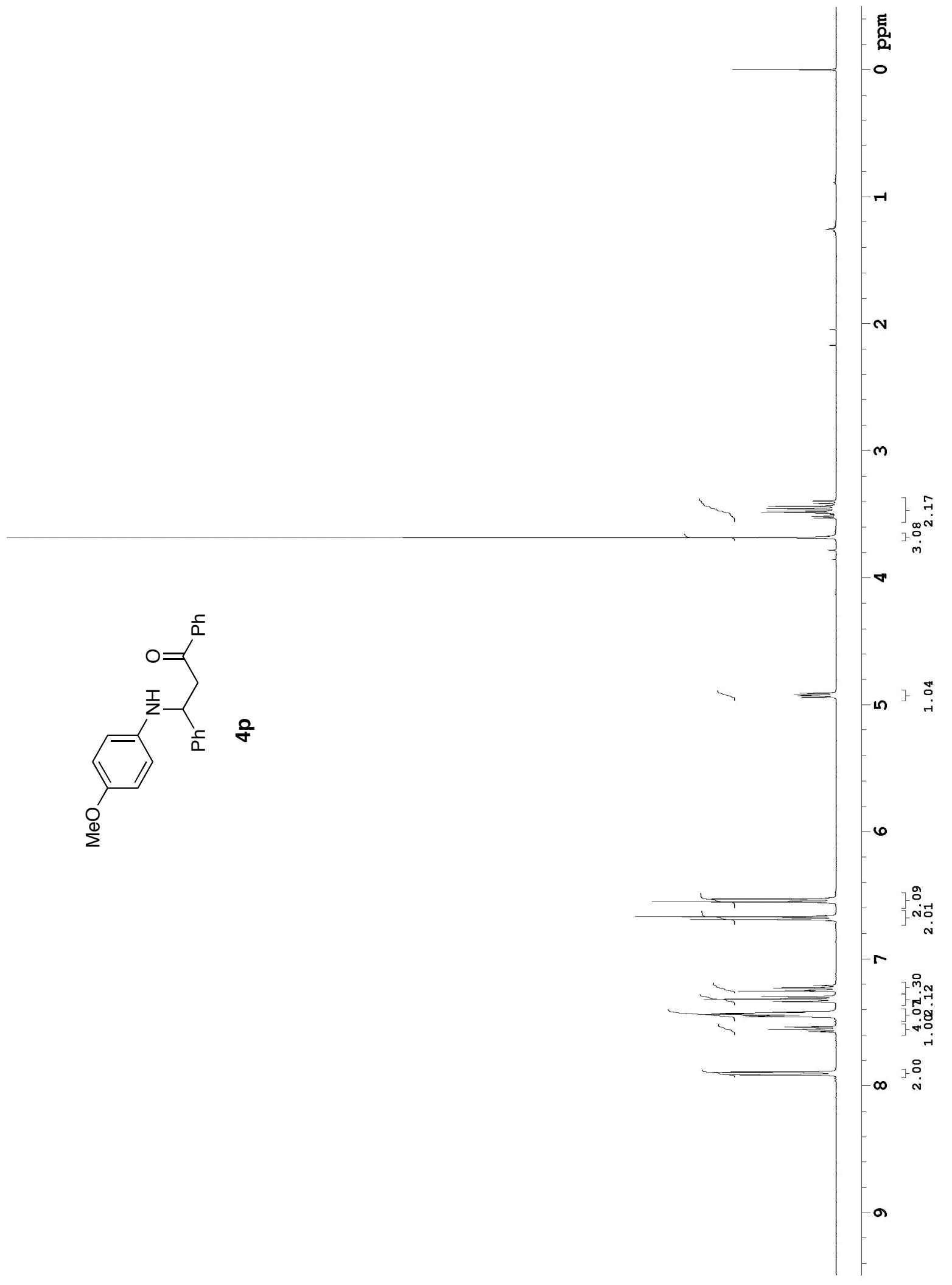



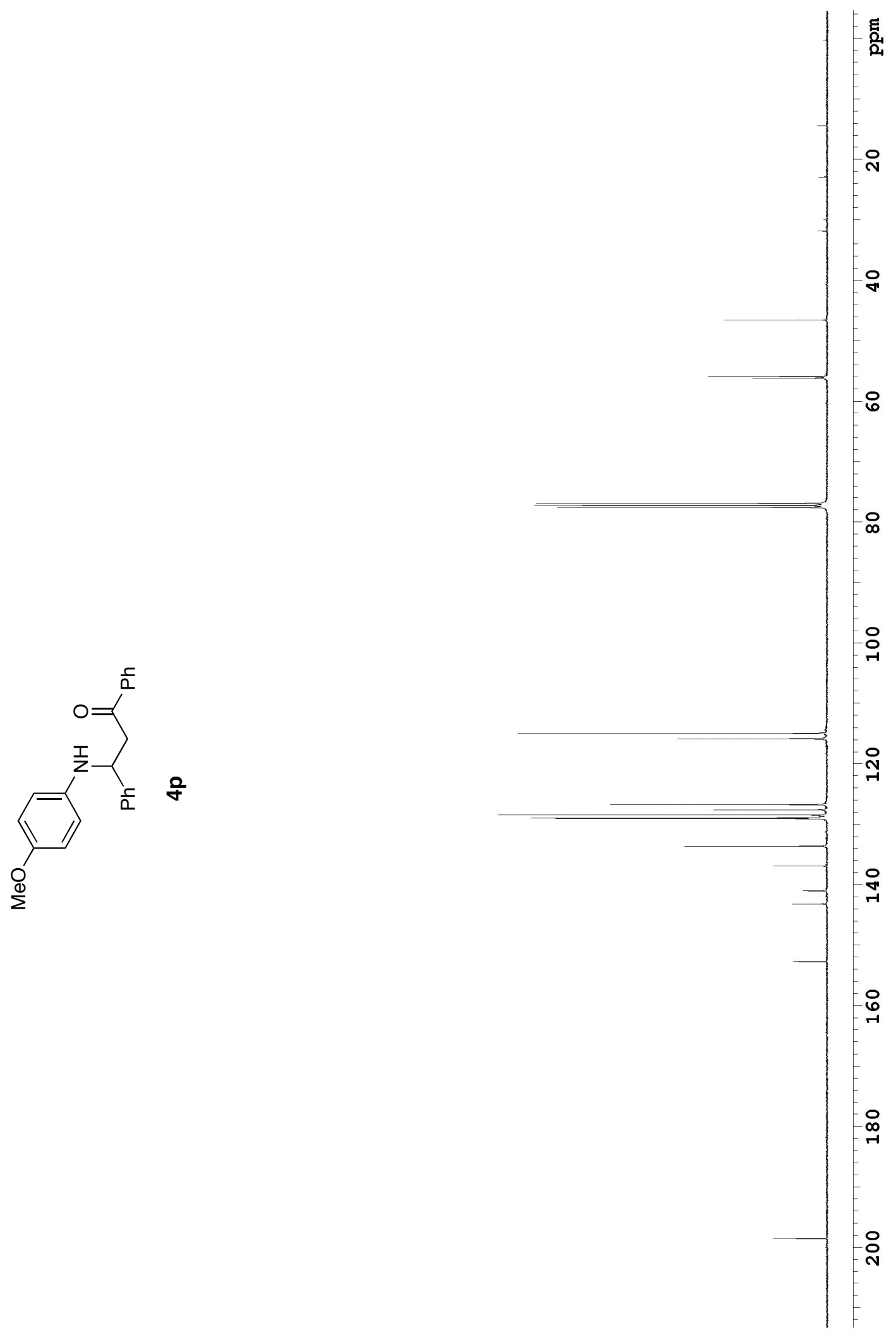

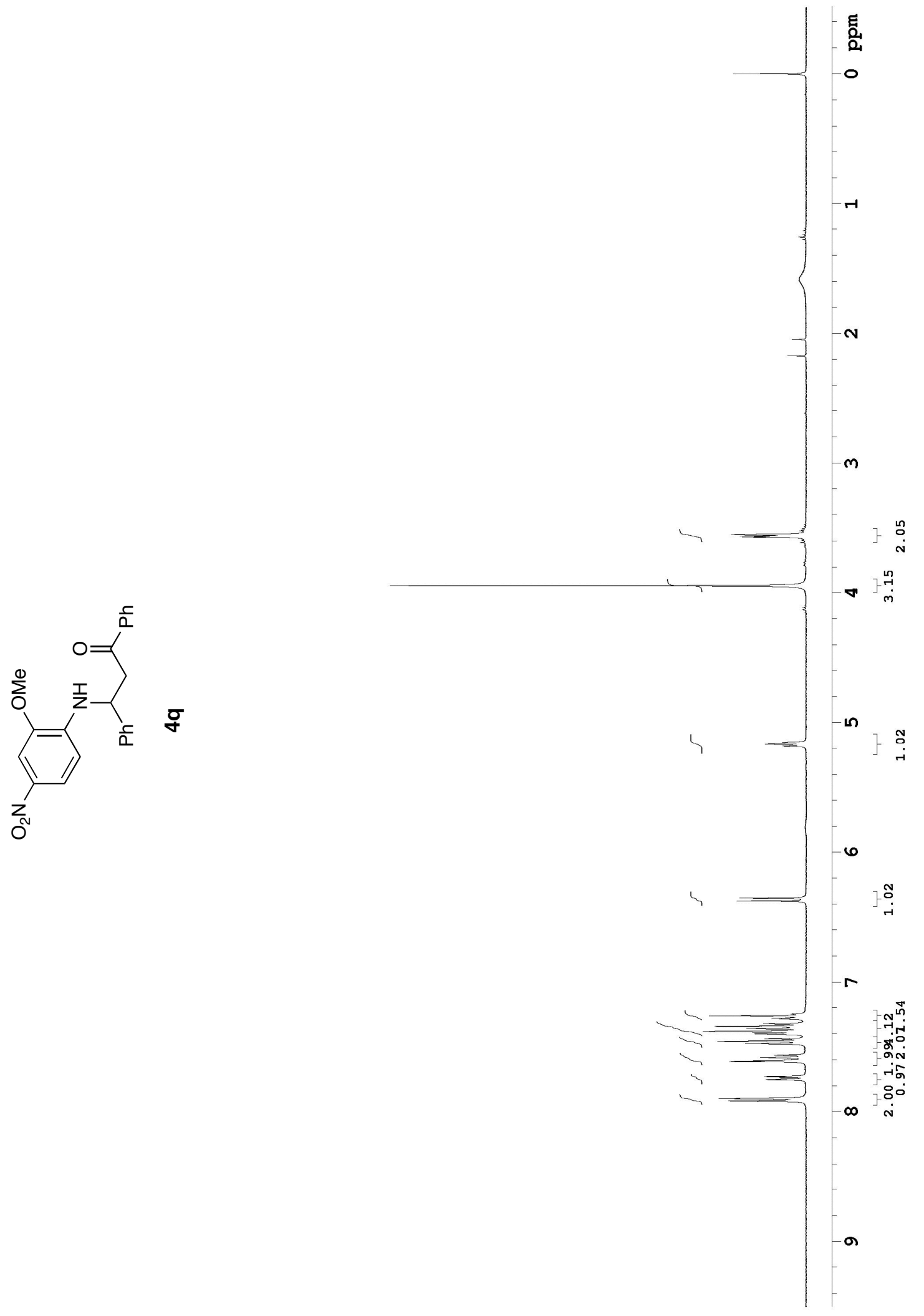


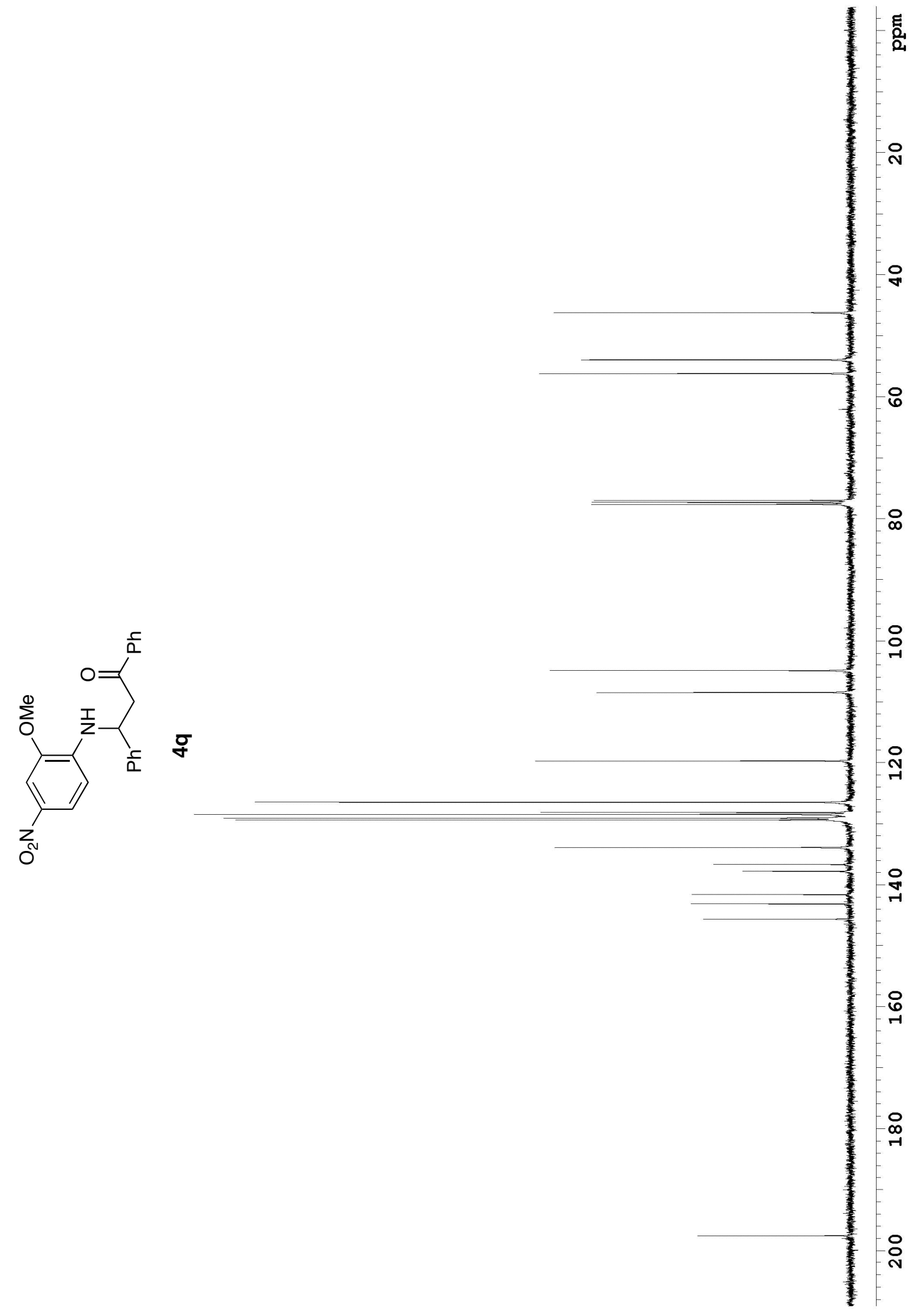




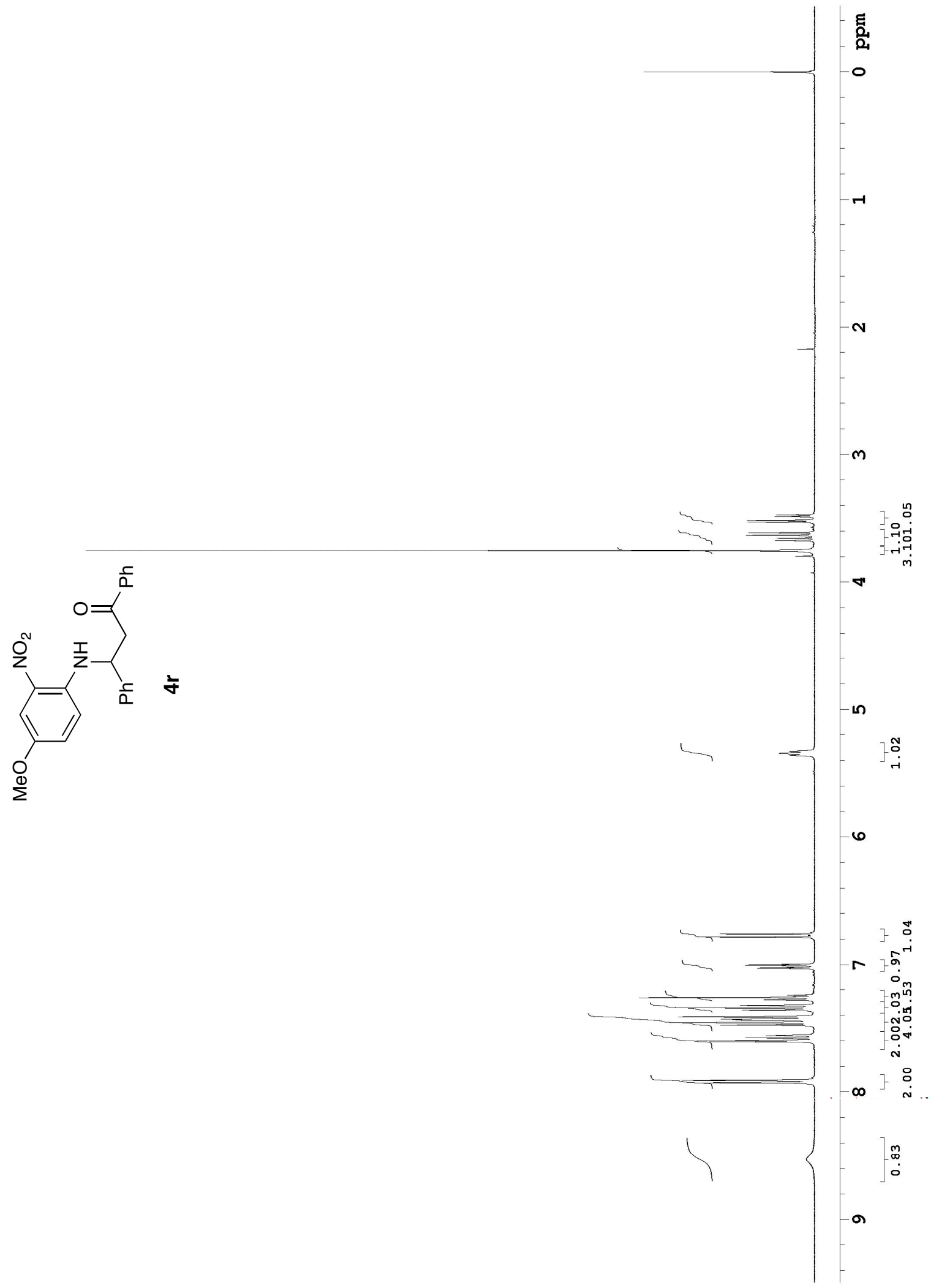




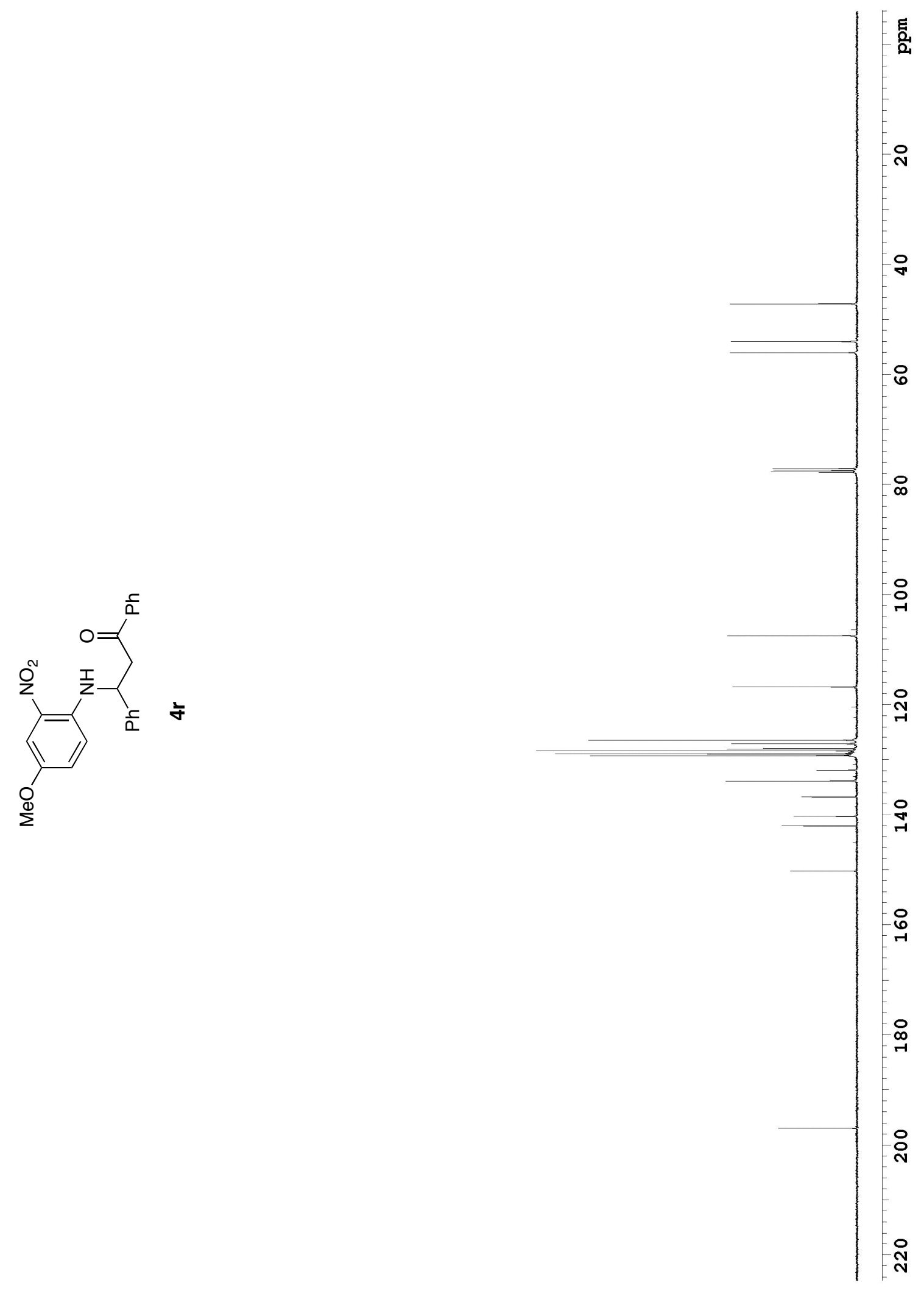




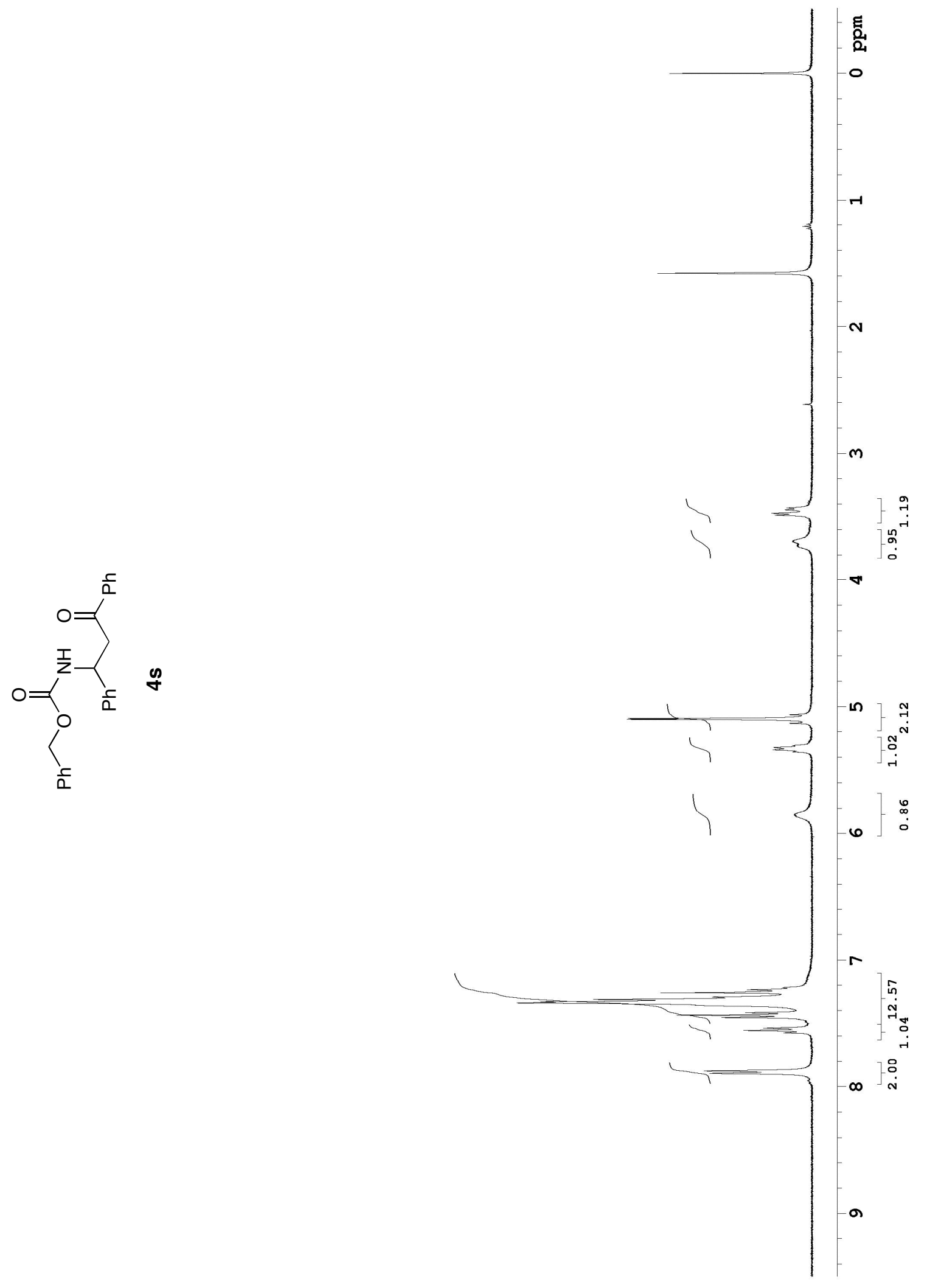




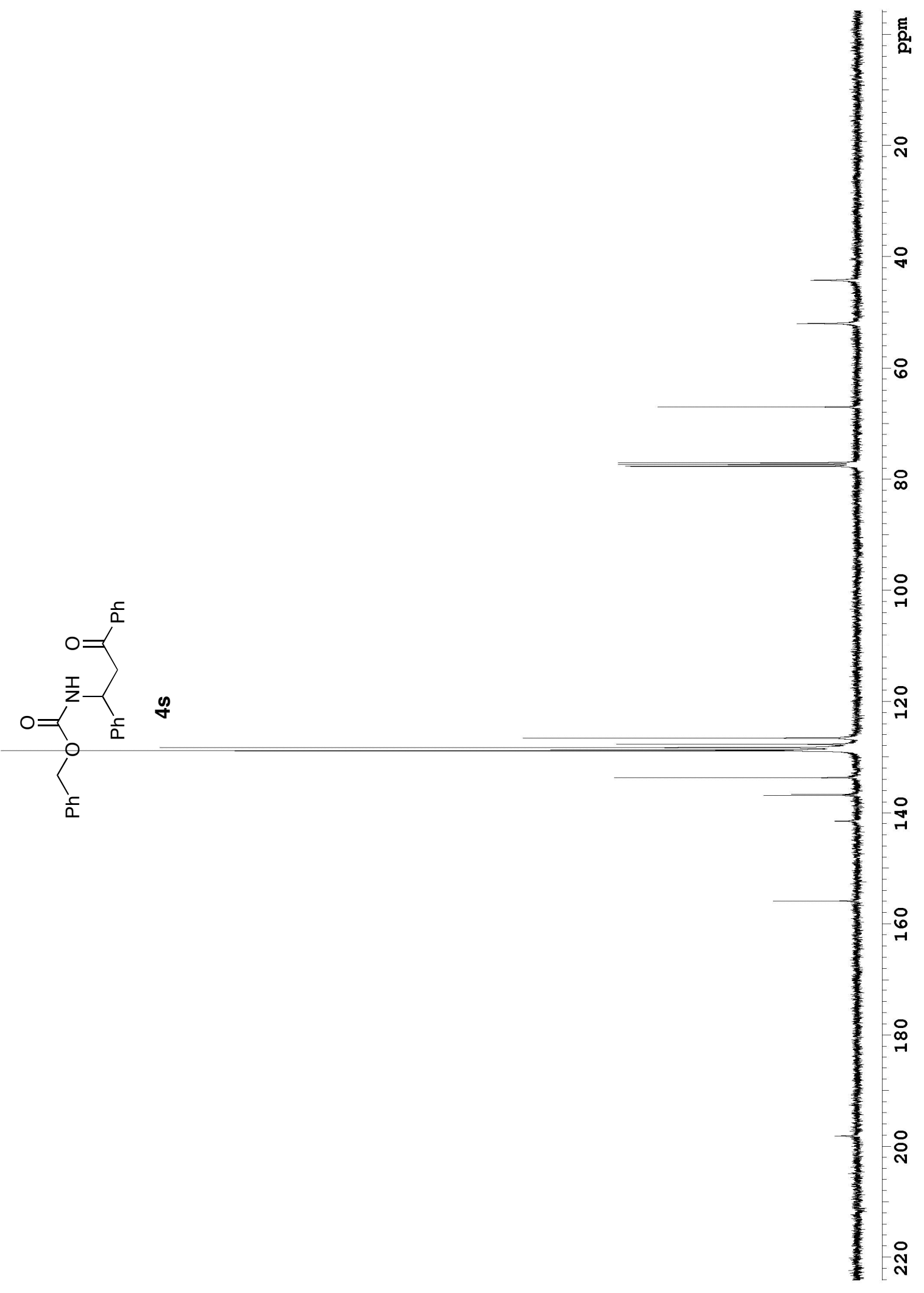

